\title{
772017
}

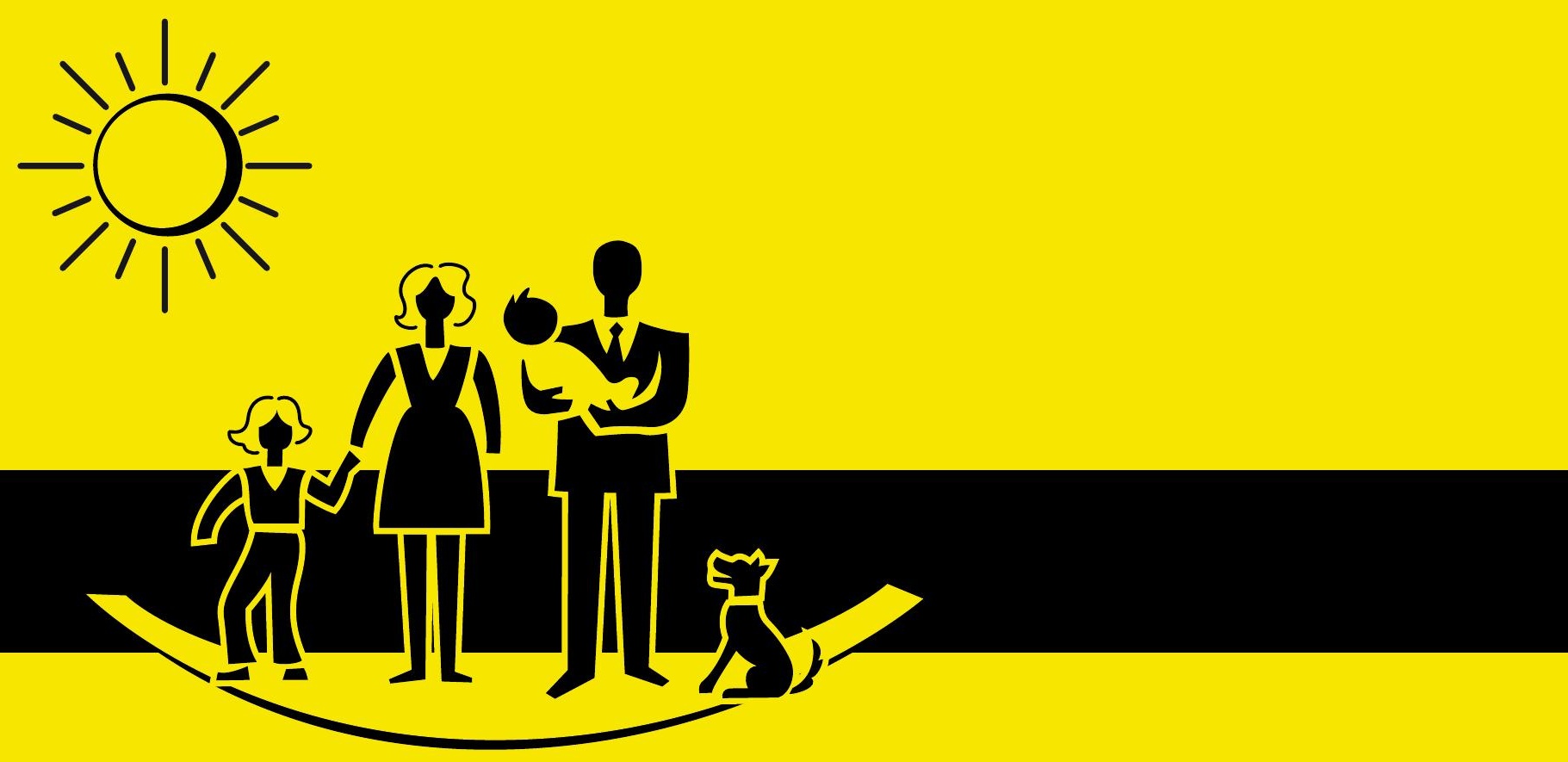

\section{Determinantes \\ sociales de la salud en Andalucía}




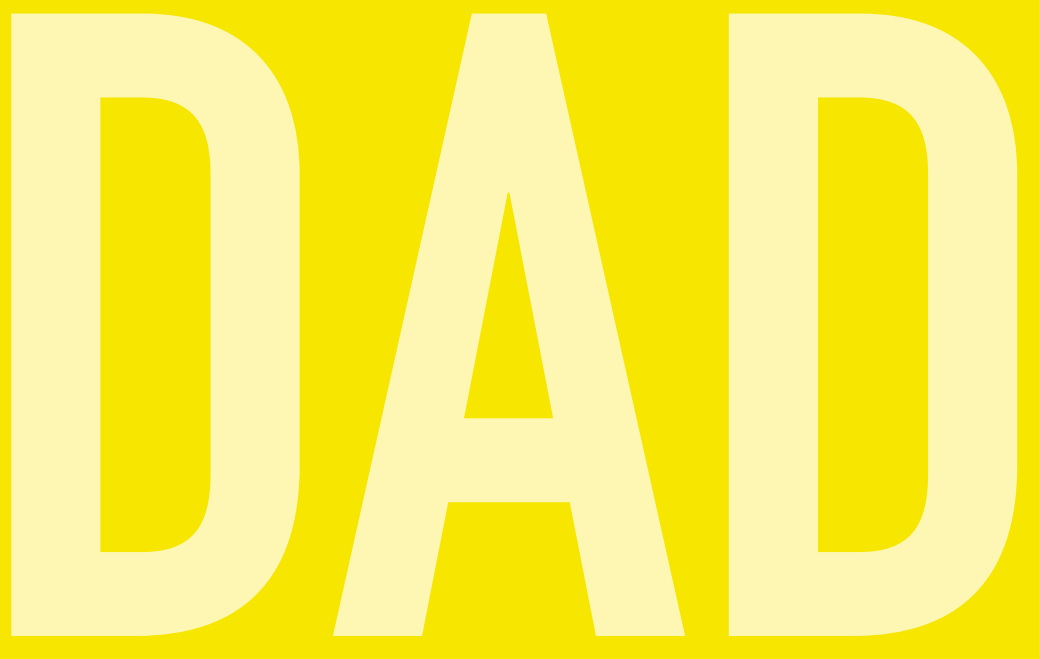


LA FUNDACIÓN CENTRO DE ESTUDIOS ANDALUCES ES UNA ENTIDAD DE CARÁCTER CIENTÍFICO Y CULTURAL, SIN ÁNIMO DE LUCRO, ADSCRITA A LA CONSEJERÍA DE LA PRESIDENCIA, ADMINISTRACIÓN LOCAL Y MEMORIA DEMOCRÁTICA DE LA JUNTA DE ANDALUCIIA. ENTRE NUESTROS OBJETIVOS FUNDACIONALES SE ESTABLECEN EL FOMENTO DE LA INVESTIGACIÓN CIENTÍFICA, LA GENERACIÓN DE CONOCIMIENTO SOBRE LA REALIDAD SOCIAL, ECONÓMICA Y CULTURAL DE ANDALUCÍA Y LA DIFUSIÓN DE SUS RESULTADOS EN BENEFICIO DE TODA LA SOCIEDAD.

NUESTRO COMPROMISO CON EL PROGRESO DE ANDALUCÍA NOS IMPULSA A LA CREACIÓN DE ESPACIOS DE INTERCAMBIO DE CONOCIMIENTO CON LA COMUNIDAD CIENTÍFICA E INTELECTUAL Y CON LA CIUDADANÍA EN GENERAL, Y A LA COLABORACIÓN ACTIVA CON LAS INSTITUCIONES PÚBLICAS Y PRIVADAS QUE INFLUYEN EN EL DESARROLLO DE LA COMUNIDAD AUTÓNOMA.

LA COLECCIÓN ACTUALIDAD FORMA PARTE DEL CATÁLOGO DE PUBLICACIONES CIENTÍFICAS DE LA FUNDACIÓN Y ESTÁ DESTINADA TANTO AL LECTOR ESPECIALIZADO COMO A LA OPINIÓN PÚBLICA EN GENERAL. CADA UNA DE SUS EDICIONES SE ESTRUCTURA COMO INFORMES MONOGRÁFICOS PARA EL FOMENTO DE LA REFLEXIÓN Y EL ANÁLISIS SOBRE ASPECTOS DE RELEVANCIA PARA LA SOCIEDAD ANDALUZA DEL SIGLO XXI.

LAS OPINIONES PUBLICADAS POR LOS AUTORES EN ESTA

COLECCIÓN SON DE SU EXCLUSIVA RESPONSABILIDAD.

(C) Del texto: los autores, 2017

(C) De la edición: Fundación Pública Andaluza

Centro de Estudios Andaluces, agosto de 2017

Bailén 50, 41001 Sevilla.

Tel.: 955055 210. Fax: 955055211

www.centrodeestudiosandaluces.es

Depósito Legal: SE-1688-05

I.S.S.N.: 1699-8294

Ejemplar Gratuito. Prohibida su venta. 



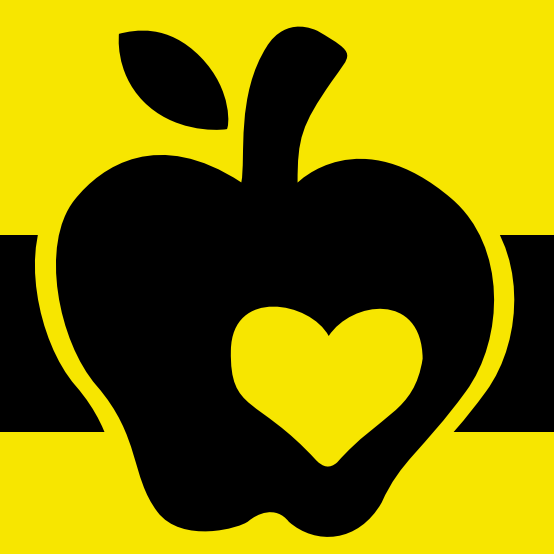

\title{
Determinantes sociales de la salud en Andalucía
}

\author{
JAVIER ÁLVAREZ GÁLVEZ \\ Univesity College London \\ Equipo investigador \\ DIEGO GÓMEZ BAYA \\ AUXILIADORA GONZÁLEZ PORTILLO \\ GLORIA MARTÍNEZ COUSINOU \\ MARÍA LUISA RODERO COSANO \\ JOSÉ ALBERTO SALINAS PÉREZ \\ EIDER MUNIATEGI AZKONA \\ Universidad Loyola Andalucía \\ Colaboradores \\ VÍCTOR SANZ SUÁREZ-LLEDÓ \\ Universidad Complutense de Madrid \\ MARÍA ISABEL SERRANO MACÍAS \\ Universidad Pablo de Olavide
}

Este documento se ha desarrollado dentro del proyecto Determinantes socioeconómicos de la salud en Andalucía: estudio comparado del impacto de la crisis económica en la salud de los andaluces (PRY120/14), financiado en la IX Convocatoria de Proyectos de Investigación del Centro de Estudios Andaluces. 


\section{ÍNDICE}

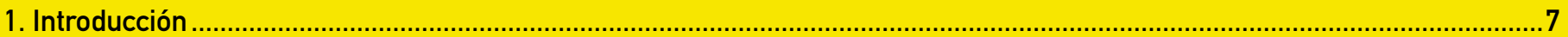

2. Aproximaciones teóricas al estudio de los determinantes sociales de la salud...............................................................

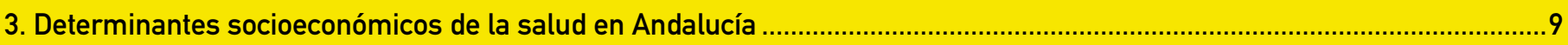

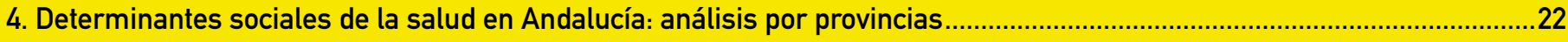

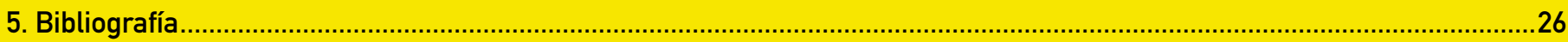




\section{Introducción}

Los determinantes sociales de la salud pueden ser definidos como aquellas condiciones sociales y económicas que pueden generar diferencias en el estado de salud y el bienestar psicosocial de los individuos y los grupos sociales a los que estos pertenecen. En un sentido amplio, son las circunstancias en que las personas nacen, crecen, viven, trabajan y envejecen (Marmot y Allen, 2014), las cuales son el resultado de la combinación de fenómenos globales complejos, como pueden ser la distribución desigual de la riqueza, el poder y los recursos, así como de las políticas adoptadas desde el ámbito global al local (Solar e Irwin, 2007). Los determinantes sociales de la salud afectan a nuestro estado físico y mental. Factores como el género, la educación, la ocupación, el nivel de ingresos, el acceso a la vivienda, el soporte social, la raza, la discapacidad o el acceso a los servicios de salud, entre otros, ejercen un poderoso efecto sobre la salud de la población, ya que a partir de estos factores se definen los modos de vida y el bienestar de las personas (Allen et al., 2014; Bartley et al., 2004; Marmot y Allen, 2014; Wilkinson, 1997). Los efectos de los determinantes sociales de la salud pueden variar de unas regiones a otras, ya que los mismos se encuentran condicionados por las diferentes políticas públicas y sociales, así como por los diferentes contextos socioculturales en los que dichos factores encuentran su marco de acción (Álvarez-Gálvez et al., 2013, 2014).

Las políticas públicas y macroeconómicas de nuestro entorno pueden afectar a las condiciones individuales en las que vivimos, determinando nuestra situación socioeconómica (Chung et al., 2013; Chung y Muntaner, 2007; Muntaner et al., 2011; Navarro, 2001), a través del establecimiento de las posibilidades de adquirir y/o mantener una posición dentro del mercado de trabajo, acceder a una vivienda digna, alcanzar un nivel educativo determinado, escalar dentro del mercado ocupacional o incluso definiendo el marco de las ayudas posibles que podemos recibir en función de nuestros ingresos, entre otros muchos factores que son susceptibles de incidir sobre la salud y el bienestar psicosocial de la ciudadanía (Solar e Irwin, 2007). La cultura en la que vivimos también ejerce un importante efecto sobre nuestra salud. Los estilos de vida y las conductas de riesgo de las personas se encuentran asociadas a la cultura y a los agentes socializadores del entorno en el que estas residen (Borg y Kristensen, 2000; Sonego et al., 2013). Llevar a cabo una vida sedentaria o el consumo de determinados alimentos de alto contenido calórico, entre otras prácticas habituales en nuestras sociedades, pueden favorecer el sobrepeso y la obesidad, por ejemplo a través del abuso de la denominada comida rápida o el elevado consumo de bebidas alcohólicas. El avance de estas conductas poco saludables no solo se encuentran vincula- das a la cultura alimentaria de un determinado país, sino también al estatus socioeconómico de la población que lo compone.

Los determinantes sociales de la salud generan desigualdades entre los diferentes grupos que componen nuestras sociedades. Las desigualdades en salud pueden ser definidas como aquellas diferencias en salud injustas y evitables entre grupos poblacionales definidos social, económica, demográfica o geográficamente (Solar e Irwin, 2007). Desigualdades que se encuentran asociadas con las menores oportunidades y recursos vinculados a la salud de personas o grupos que presentan un conjunto determinado de características (sexo, edad, estatus socioeconómico, etnia o lugar de residencia, entre otras) que pueden afectar negativamente a su salud y bienestar (Whitehead, 1992). Ahora bien, las desigualdades de salud no solo afectan a los países en vías de desarrollo. De hecho, recientes estudios demuestran que las desigualdades de salud también persisten dentro del marco de países económicamente avanzados con acceso a sistemas de salud ampliamente desarrollados (Bambra et al., 2010, 2014; Álvarez-Gálvez, 2014, 2016; Mackenbach, 2012). En el marco europeo, en función de las diferentes regiones, se pueden apreciar enormes diferencias en indicadores básicos de salud como pueden ser la mortalidad o la esperanza de vida (Jakab y Marmot, 2014). Por ejemplo, la esperanza de vida de los hombres en Rusia se encuentra 20 puntos por debajo de la de Islandia; la esperanza de vida de las personas de 30 años con estudios se encuentra 5 puntos por encima de sus homólogos con estudios secundarios. Tener estudios superiores, unos ingresos elevados y/o una elevada cualificación profesional puede incrementar las probabilidades individuales de tener buena salud y este hecho se puede aplicar a cualquier contexto geográfico.

Por consiguiente, la riqueza material de un país o región determinada no debe llevarnos a suponer la ausencia de desigualdades en términos sanitarios, ya que los determinantes sociales de la salud son múltiples y diversos en función de los diferentes contextos analizados. Asimismo, dependiendo de las políticas adoptadas y las diferencias socioculturales de los diferentes territorios, el efecto de estos factores puede variar de una región a otra. En este sentido, nuestra salud se encuentra próximamente vinculada al contexto económico, político, social y cultural en el que vivimos. Sin embargo, aunque existe un relativo acuerdo en torno a los efectos de los diferentes determinantes sociales de la salud, dentro de la literatura también es posible encontrar diferentes teorías que tratan de poner de manifiesto los mecanismos inherentes de estos procesos de interacción entre factores sociales y sanitarios, así como las posibles explicaciones a la (re)producción de desigualdades de salud. 


\section{Aproximaciones teóricas al estudio de los determinantes sociales de la salud}

Dentro del ámbito de estudio de los determinantes sociales de la salud podemos identificar tres aproximaciones principales: (1) la teoría psicosocial; (2) la teoría sobre la producción social de la enfermedad o economía política de la salud y (3) la teoría ecosocial. Aunque cada una de estas tres aproximaciones teóricas dan cuenta de diferentes explicaciones posibles al desarrollo de desigualdades en salud, en la práctica todas mantienen un cierto grado de interconexión, y, al mismo tiempo, utilidad para la comprensión de los diferentes mecanismos y procesos implicados en estos fenómenos complejos (Solar e Irwin, 2007).

La teoría psicosocial sobre los determinantes sociales de la salud pone el foco de atención, como su nombre claramente indica, sobre los factores psicosociales (Raphael, 2006; Raphael y Bryant, 2006). En concreto, esta teoría se centra en explicar cómo los individuos perciben y evalúan su propio estatus personal en el marco de jerarquías sociales y sociedades desiguales, una experiencia que puede conducir al desarrollo de estrés y en ocasiones a una peor salud (Wilkinson, 2000; Marmot y Wilkinson, 2001; Wilkinson y Pickett, 2006). Esta idea deriva del estudio de Cassel (Cassel, 1976), en el que se indica que el estrés del «ambiente social» puede afectar a la susceptibilidad del individuo, y por lo tanto, a sufrir alteraciones neuroendocrinas que pueden aumentar la vulnerabilidad del organismo hacia el desarrollo de determinadas enfermedades. Desde este punto de vista, es la propia autopercepción del individuo, el modo en el que se ve frente a los demás en la jerarquía social, la que puede desencadenar el desarrollo de sentimientos negativos y la desintegración de vínculos sociales que, en última instancia, pueden dar paso a una peor salud mental y física (Lobmayer y Wilkinson, 2002; Lynch et al., 2001; Marmot, 2002; Marmot y Wilkinson, 2001; Wilkinson, 2000; Wilkinson y Pickett, 2006).

En un segundo lugar, la teoría de la producción social de la enfermedad o teoría de la economía política de la salud, se centra en la explicación de los determinantes económicos y políticos de la salud (Kaplan et al., 1996), también conocida como la aproximación materialista o neo-materialista en el estudio de los determinantes sociales de la salud. Los autores de esta perspectiva teórica están de acuerdo en el impacto de los factores sociales sobre la salud, aunque no consideran que estos sean la causa principal del problema. A diferencia de los autores de la teoría psicosocial, los de esta perspectiva teórica ponen el foco en las causas estructurales de las desigualdades sociales y de la salud. En otras palabras, podemos decir que el origen del problema se encuentra en la distribución desigual de recursos económicos y sociales, generados por la interacción entre los procesos económicos del mercado y las políticas públicas del estado (políticas sociales, sanitarias, educativas, medio-ambientales, en materia de vivienda, etc.), y no en la percepción de los individuos (Kaplan et al., 1996; Lynch et al., 1998).

En tercer lugar, la teoría ecosocial integra las dos aproximaciones teóricas anteriores dentro de un mismo marco. La aproximación ecosocial al estudio de los determinantes sociales de la salud combina en un enfoque multinivel los factores de carácter biológico y social, a través de una perspectiva dinámica y compleja, que tiene en cuenta las causas históricas y ecológicas que dan lugar a las desigualdades de salud. Desde esta perspectiva teórica se parte de la idea de que las personas incorporan biológicamente sus experiencias de desigualdad social desde la vida intrauterina hasta la muerte (Krieger, 2005). Dicho de otro modo, podemos decir que desde este enfoque, la biología de las personas se encuentra determinada no solo por las circunstancias vividas a lo largo del ciclo vital, sino incluso por las circunstancias previas a su propia concepción a nivel celular. En este sentido, las condiciones de vida de nuestros ascendientes juegan un papel esencial en nuestra salud futura. El hecho de tener unos padres que hayan sufrido condiciones de desigualdad social y económica, por ejemplo, puede dar lugar a unas peores pautas alimenticias (dietas bajas en vitaminas o elevadas en grasas nocivas) 0 incluso a algunas conductas de riesgo (consumo de alcohol, tabaco, etc.) que posteriormente podrían afectar a nuestra biología y al desarrollo de nuestra vida futura (Krieger, 2005, 2001). Así, incorporamos las influencias biológicas a partir de las influencias del mundo material y social en el vivimos, una dinámica que Krieger (Krieger, 2005) define como proceso de «encarnación» (o embodiment en inglés).

Las denominadas teorías del ciclo vital incorporan el estudio de los procesos de movilidad social al estudio de las desigualdades de salud (Benzeval y Judge, 2001), asumiendo que los cambios inter e intrageneracionales en la posición socioeconómica de los individuos, (esto es, en su clase social), explican los cambios en la salud (Illsley, 1955; West, 1991), al mismo tiempo que las variaciones en la salud pueden afectar a los procesos de movilidad social (Bartley et al., 1994; Blane et al., 1999; Illsley, 1955; West, 1991). Sin embargo, las actuales evidencias presentan resultados contradictorios. 
Los procesos de movilidad social descendentes pueden favorecer la salud al igual que la movilidad ascendente puede empeorarla. En relación a estos resultados, se han encontrado explicaciones que apuntan a fenómenos de «resiliencia» —entendida como la capacidad del individuo para superar circunstancias traumáticas - entre personas que caen en procesos de movilidad descendente (esto es, que ven empeorar sus condiciones materiales de vida), 0 al estrés que puede generar el llegar a una clase superior en el caso de los procesos de movilidad ascendente (Blane et al., 1999; Davey Smith et al., 1998; Hart et al., 1998; Power et al., 2002). En todo caso, todavía no existe una explicación clara en este sentido, la complejidad y el dinamismo de estos procesos de influencia siguen dificultando la obtención de unos resultados que sean concluyentes (Alvarez-Galvez, 2016; Beckfield y Krieger, 2008; Mackenbach, 2012).

Tratando de arrojar algo de luz a estas cuestiones, el presente trabajo se dirige al estudio de los determinantes de la salud en Andalucía a través de la explotación de los resultados de una encuesta desarrollada durante los meses de enero a abril de 2016 en el marco del proyecto IMPACT-A: Determinantes socioeconómicos de la salud en Andalucía: estudio comparado del impacto de la crisis económica en la salud de los andaluces (PRY120/14), financiado por la IX Convocatoria de Proyectos de Investigación del Centro de Estudios Andaluces. En total, fueron entrevistadas 1.200 personas mayores de 18 años y residentes en las ocho provincias andaluzas. Con la intención de comprender el posible impacto de la crisis económica sobre la salud de los andaluces y andaluzas, se analizaron diferentes cuestiones que hacían referencia a las dificultades económicas experimentadas durante el periodo de crisis económica, la percepción de los efectos de la crisis económica sobre la salud de los mismos, las necesidades sanitarias, y el uso de los servicios de salud entre la población andaluza.

\section{Hasta un $70,4 \%$ de los andaluces mayores de 65 años consumen dos o más veces al día fruta, mientras que este porcentaje se reduce al $36,6 \%$ en el segmento de 18-34 años}

\section{Determinantes socioeconómicos de la salud en Andalucía}

\subsection{Estilos de vida de la población andaluza}

En las figuras 1-5 se describen los estilos de vida de la población andaluza, por género y edad, en concreto del: (1) consumo de frutas; (2) consumo de vegetales y ensaladas; (3) práctica de actividad física; (4) consumo de tabaco, y (5) consumo de alcohol. Respecto al consumo de frutas, más de la mitad de la población andaluza (51\%) toma fruta dos veces o más al día, mientras que sólo un 5 \% nunca toma fruta o lo hace menos de una vez a la semana. Las mujeres toman más fruta que los hombres, de manera que el $57,2 \%$ toman dos o más veces fruta al día frente al 44,6\% de los hombres. También se han hallado diferencias de edad relevantes. Hasta un 70,4 \% de los andaluces mayores de 65 años consumen dos o más veces al día fruta, mientras que este porcentaje se reduce al 36,6 \% en el segmento de 18-34 años. En la figura 1 se representan los porcentajes de consumo de fruta por género y edad.

Respecto al consumo de vegetales y ensaladas frescas, dos terceras partes de los andaluces toma al menos una vez al día vegetales o ensaladas $(65,9 \%)$, aunque casi una quinta parte de los andaluces $(19,1 \%)$ consume vegetales 0 ensaladas menos de 4 veces a la semana o nunca. Nuevamente se ha encontrado que las mujeres toman más vegetales que los hombres, de manera que el 29,7\% de las mujeres consumen vegetales dos o más veces al día, frente al 15,7 \% de los hombres. También se dan diferencias según la edad, de forma que los andaluces entre 35-49 años son los que más vegetales toman (un 27,5 \% toman vegetales dos veces o más al día) en comparación con los menores de 34 años (sólo un 17,7 \% consumen vegetales dos veces o más al día). En la figura 2 se representan los porcentajes por género y edad del consumo de vegetales y ensaladas frescas.

Con referencia a la práctica de actividad físico-deportiva, los andaluces practican casi 3 días a la semana algún tipo de actividad física o deporte, como es caminar rápidamente $(M=2,82, S D=2,63)^{1}$. Los hombres $(M=3,10$,

${ }^{1}$ M: mean (media); SD: standard deviation (desviación estándar). 


\section{Figura 1. Frecuencia de consumo de fruta, por género y edad}

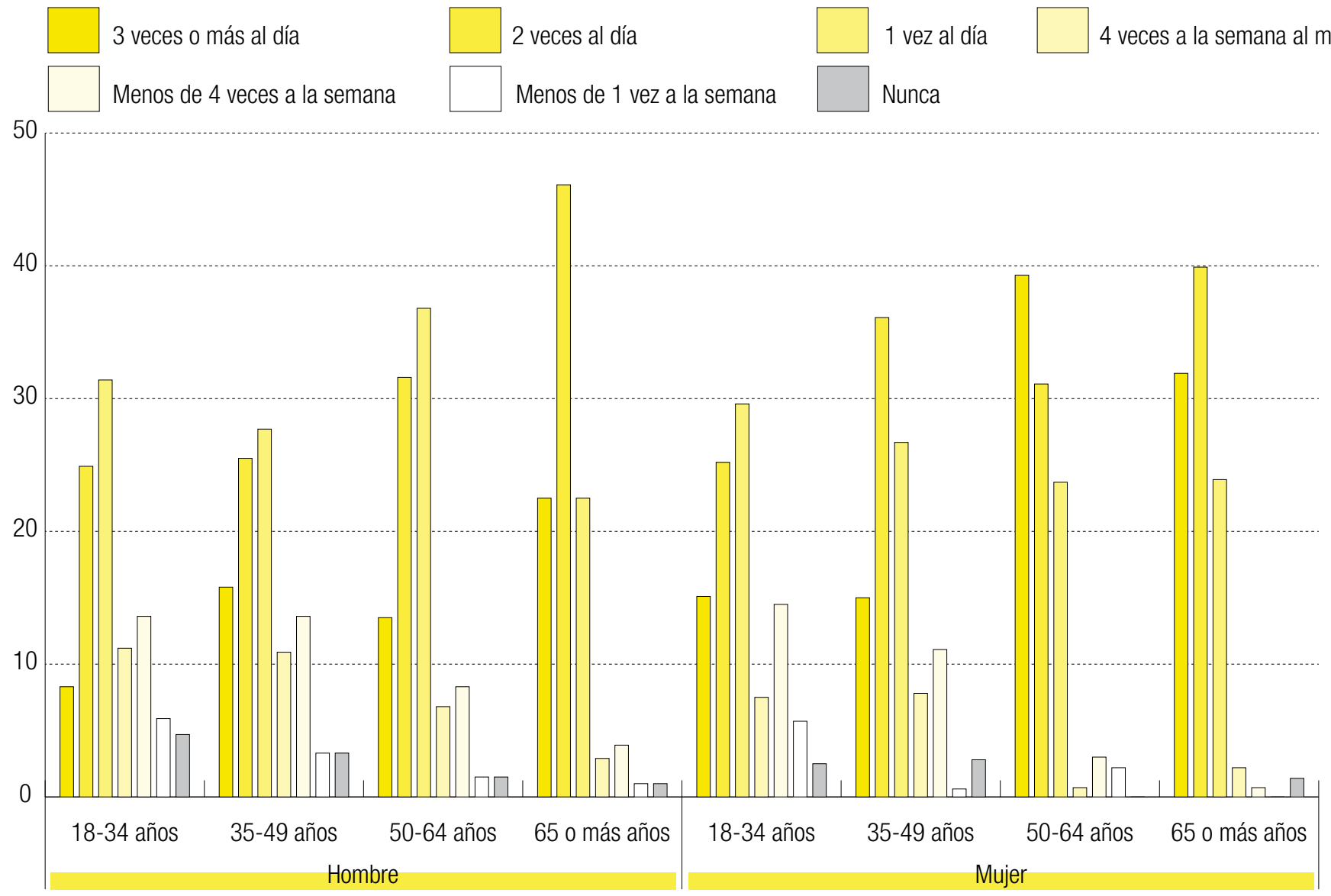

Fuente: elaboración propia a partir de los datos de la encuesta del Proyecto IMPACT-A.

$\mathrm{SD}=2,66)$ practican más actividad físico-deportiva que las mujeres $(\mathrm{M}=2,55$, $\mathrm{SD}=2,56)$. No se encuentran diferencias significativas en función de la edad. Respecto a las diferencias de género, las más destacables se encuentran en el segmento de mayor edad, en el que los hombres hacen actividad físicodeportiva por término medio más de un día a la semana más que las mujeres (figura 3).

Con respecto al consumo de tabaco, un 48,3 \% de los andaluces declaran que nunca han fumado, y un 28,8 \% lo hicieron pero lo han dejado. De los que fuman, el 22,8 \% restante, casi la tercera parte (un 8,9\% del total de la muestra) fuma entre 11 y 20 cigarrillos al día. Se han encontrado diferencias de género destacables, de manera que un $54,6 \%$ de las mujeres nunca ha fumado, frente al $41,8 \%$ de los hombres. Entre los varones, un 11,7 \% fuma
11-20 cigarrillos diarios frente al 6,2 \% de las mujeres. Se han encontrado también diferencias en función de la edad, de forma que 27,7\% de los andaluces entre 18-34 años fuman, el 25,9\% en el caso de los andaluces de 35-49 años, el 24,6 \% de los andaluces entre 50-64 años y el 9,6 \% de mayores de 65 años. Resulta destacable que un 39,2 \% de los andaluces entre 50-64 años ha dejado de fumar, mientras que el 19,9\% de los menores de 34 años fuman entre 1-10 cigarrillos diarios. En la figura 4 podemos observar que alrededor del $15 \%$ de los varones de 35-65 años fuman entre 11 y 20 cigarrillos al día, mientras que este porcentaje está alrededor del $8 \%$ en las mujeres de esas edades.

Finalmente, en la figura 5 se representa el consumo de alcohol por género y edad. Casi una cuarta parte de los andaluces toma bebidas alcohólicas 

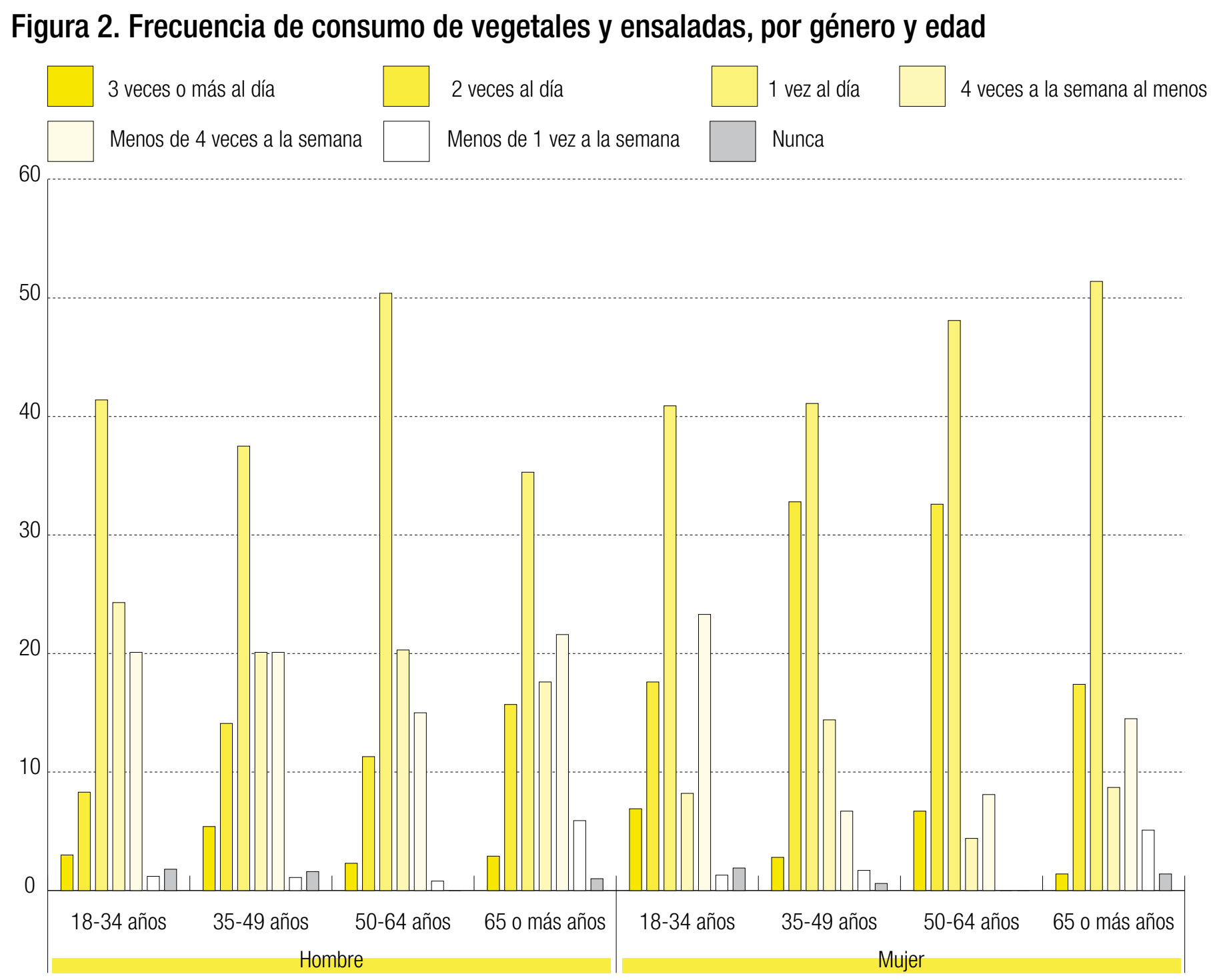

Fuente: elaboración propia a partir de los datos de la encuesta del Proyecto IMPACT-A.

(23,9 \%) varias veces a la semana o todos los días, mientras que un $36,8 \%$ nunca toma estas bebidas. Los varones consumen con más frecuencia bebidas alcohólicas que las mujeres, de forma que un 37,6 \% bebe todos los días o varias veces a la semana, frente a un 10,8 \% de las mujeres. Entre las mujeres, un 47,9\% nunca toma bebidas alcohólicas, mientras que este porcentaje es del $25,3 \%$ en la muestra de hombres. También se destacan diferencias de edad. Los mayores de 65 años nunca beben en un 58,3 \%, frente al 26,2 \% de los jóvenes de entre 18-34 años. En la figura 5 se des- taca que entre los andaluces de más de 65 años, las mujeres nunca beben en un $77,5 \%$ de los casos, mientras que en el caso de los hombres, beben diariamente en un $48 \%$. El 43,8 \% de los varones de 18-34 años toma alcohol varias veces o una vez a la semana, frente al $26,4 \%$ de las mujeres de esa edad. 
Figura 3. Media de días en la última semana que caminó rápidamente o practicó algún tipo de deporte 0 actividad física durante al menos 30 minutos, por género y edad

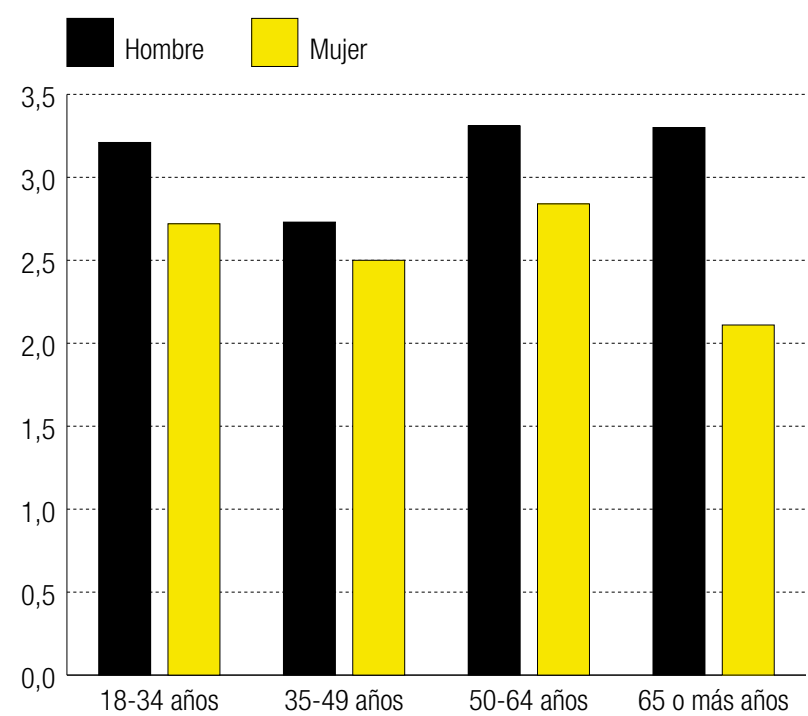

Casi una cuarta parte de los andaluces toma bebidas alcohólicas $(23,9 \%)$ varias veces a la semana o todos los días, mientras que un $36,8 \%$ nunca toma estas bebidas; los varones consumen con más frecuencia bebidas alcohólicas que las mujeres, de forma que un $37,6 \%$ bebe todos los días 0 varias veces a la semana, frente a un $10,8 \%$ de las mujeres

Fuente: elaboración propia a partir de los datos de la encuesta del Proyecto IMPACT-A.

Figura 4. Consumo de tabaco, por género y edad

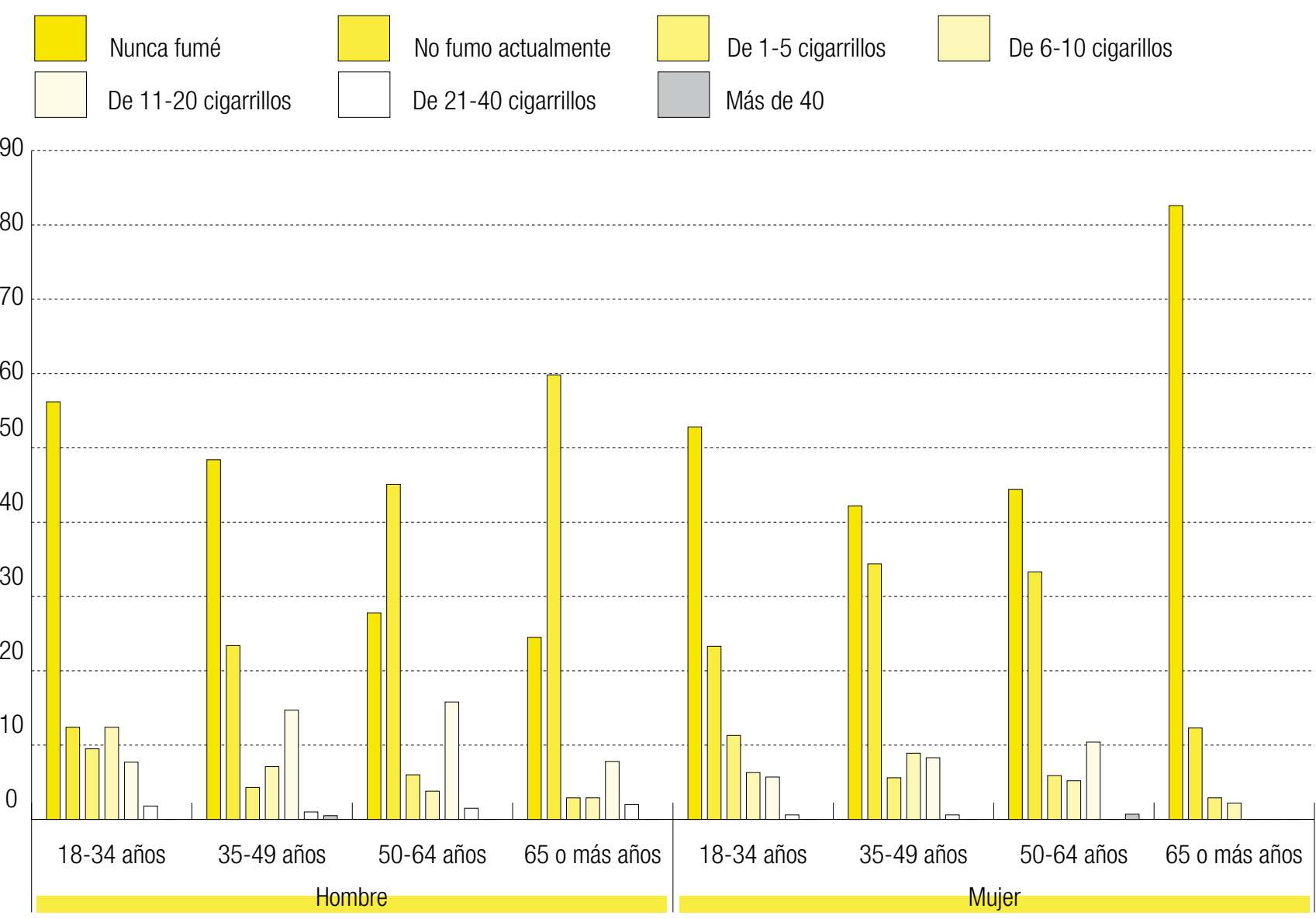


Figura 5. Consumo de bebidas alcohólicas, por género y edad

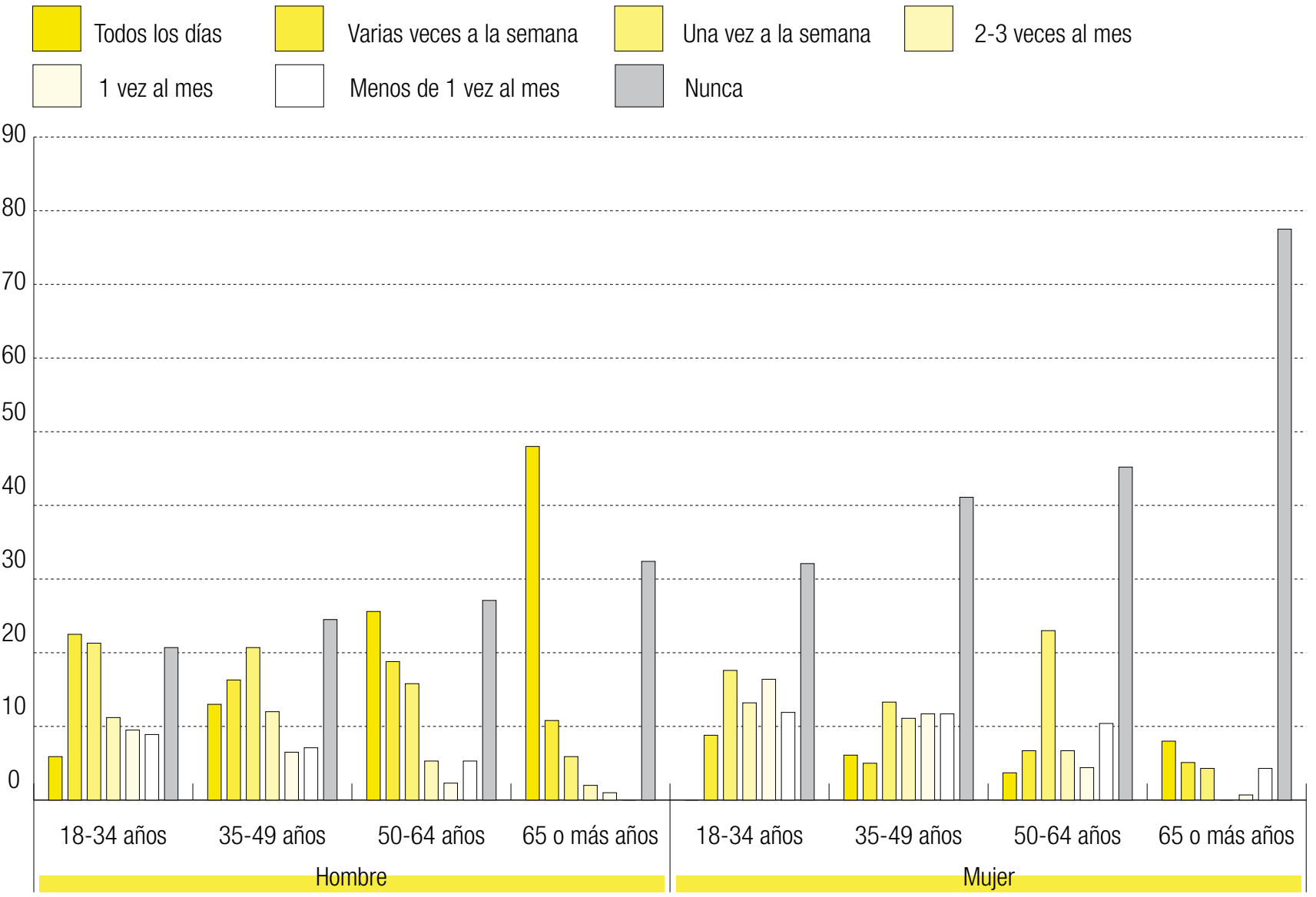

Fuente: elaboración propia a partir de los datos de la encuesta del Proyecto IMPACT-A. 


\subsection{Diferencias en el uso de servicios de salud}

En la tabla 1 se describe el uso de los servicios de salud tanto públicos como privados en función del sexo, la edad, el grupo de ingresos (medido por cuartiles), el nivel educativo y la provincia de residencia. Como se puede apreciar en la tabla, las mujeres andaluzas usan en mayor medida los servicios de atención primaria y los servicios especializados. El $65 \%$ de las mujeres entrevistadas admitían haber acudido al médico de cabecera para su atención personal, frente al 59,4 \% de los hombres. Del mismo modo, un $45,9 \%$ de las mujeres había acudido a algún servicio de carácter especializado frente al 37,8 \% de los hombres. A su vez, las andaluzas también se encuentran por encima de los andaluces en el uso de servicios de urgencia y en el porcentaje de ingresos hospitalarios, aunque estas diferencias no resultan estadísticamente significativas $(p>0,05)$. Por grupos de edad, suelen ser los grupos mayores los que, en general, hacen un mayor uso de los servicios de salud. En este caso, encontramos diferencias estadísticamente significativas en el uso de servicios de urgencia y los especializados, en los cuales también podemos ver unos porcentajes relativamente elevados entre los grupos más jóvenes.

A partir de los datos extraídos, el nivel de ingresos y la educación no parecen ser factores determinantes en el uso de los servicios de salud en Andalucía, aunque se observan algunas diferencias en los porcentajes que ponen de manifiesto que las personas con menores ingresos hacen un mayor uso de los servicios de atención primaria frente a los especializados. Por ejemplo, el $65 \%$ de las personas situadas en el tramo de ingresos más bajos (C1) hicieron uso de estos servicios frente al 56,1 \% del grupo con mayores ingresos. Mientras que, a la inversa, los grupos de mayores ingresos hacían mayor uso de servicios especializados (46,0 \%) frente al grupo con menores ingresos (38,5\%). La educación no presenta una relación estadísticamente significativa con el uso de los servicios de salud, aunque muestra una cierta tendencia hacia la significatividad en los ingresos hospitalarios entre los grupos con estudios de primaria y universitarios. Finalmente, tampoco se encuentran grandes diferencias en función de la provincia de residencia, de hecho solo se detectan diferencias estadísticamente significativas para el uso de los servicios de urgencia, en las cuales los residentes en Málaga (38,7 \%), Almería (38,0 \%) y Cádiz (31,5\%) son los que parecen hacer un mayor uso de estos servicios.

\section{Tabla 1. Uso de servicios de salud de la población andaluza}

\begin{tabular}{lllll} 
& $\begin{array}{l}\text { Servicios } \\
\text { de atención } \\
\text { primaria }\end{array}$ & $\begin{array}{l}\text { Servicios } \\
\text { de urgencia }\end{array}$ & $\begin{array}{l}\text { Ingreso } \\
\text { hospitalario }\end{array}$ & $\begin{array}{l}\text { Servicios } \\
\text { de atención } \\
\text { especializada }\end{array}$ \\
\hline Hombre & $59,4 \%$ & $28,0 \%$ & $13,4 \%$ & $37,8 \%$ \\
Mujer & $65,5 \%$ & $31,9 \%$ & $14,9 \%$ & $45,9 \%$ \\
Sig. & 0,028 & 0,143 & 0,694 & 0,004 \\
18-29 & $61,3 \%$ & $36,5 \%$ & $10,5 \%$ & $31,4 \%$ \\
30-39 & $60,2 \%$ & $33,5 \%$ & $12,6 \%$ & $40,9 \%$ \\
40-49 & $62,2 \%$ & $25,5 \%$ & $10,9 \%$ & $37,5 \%$ \\
50-59 & $61,1 \%$ & $25,4 \%$ & $12,2 \%$ & $43,8 \%$ \\
60-69 & $65,2 \%$ & $23,9 \%$ & $21,2 \%$ & $50,4 \%$ \\
70-79 & $64,4 \%$ & $30,8 \%$ & $25,0 \%$ & $47,5 \%$ \\
80 o más & $72,9 \%$ & $42,4 \%$ & $16,0 \%$ & $57,6 \%$ \\
Sig. & 0,646 & 0,014 & 0,382 & 0,002 \\
C1 & $65,0 \%$ & $33,7 \%$ & $14,7 \%$ & $38,5 \%$ \\
C2 & $66,0 \%$ & $29,3 \%$ & $11,8 \%$ & $42,0 \%$ \\
C3 & $61,5 \%$ & $30,2 \%$ & $14,7 \%$ & $43,5 \%$ \\
C4 & $56,1 \%$ & $22,3 \%$ & $24,2 \%$ & $46,0 \%$ \\
Sig. & 0,190 & 0,103 & 0,427 & 0,418 \\
Primaria & $71,7 \%$ & $39,1 \%$ & $27,8 \%$ & $45,7 \%$ \\
Secundaria $\%$ & $63,0 \%$ & $29,9 \%$ & $11,0 \%$ & $40,5 \%$ \\
Universidad & $62,8 \%$ & $29,3 \%$ & $15,7 \%$ & $40,4 \%$ \\
Postgrado & $60,0 \%$ & $28,8 \%$ & $11,3 \%$ & $44,0 \%$ \\
Sig. & 0,216 & 0,257 & 0,063 & 0,605 \\
Almería & $65,1 \%$ & $38,0 \%$ & $8,8 \%$ & $42,3 \%$ \\
Cádiz & $68,5 \%$ & $31,5 \%$ & $14,9 \%$ & $38,9 \%$ \\
Córdoba & $70,0 \%$ & $26,9 \%$ & $17,5 \%$ & $44,0 \%$ \\
Granada & $57,3 \%$ & $27,3 \%$ & $12,2 \%$ & $48,0 \%$ \\
Huelva & $60,0 \%$ & $25,3 \%$ & $18,4 \%$ & $37,3 \%$ \\
Jaén & $58,0 \%$ & $26,7 \%$ & $7,5 \%$ & $36,0 \%$ \\
Málaga & $59,3 \%$ & $38,7 \%$ & $15,5 \%$ & $47,7 \%$ \\
\hline Sevilla & $62,0 \%$ & $25,3 \%$ & $21,1 \%$ & $41,3 \%$ \\
\hline & 0,170 & 0,033 & 0,603 & 0,284
\end{tabular}

Fuente: elaboración propia a partir de los datos de la encuesta del Proyecto IMPACT-A. 


\section{Figura 6. Uso de servicios de salud de atención primaria, urgencias y especializados de la población andaluza en función de los ingresos}

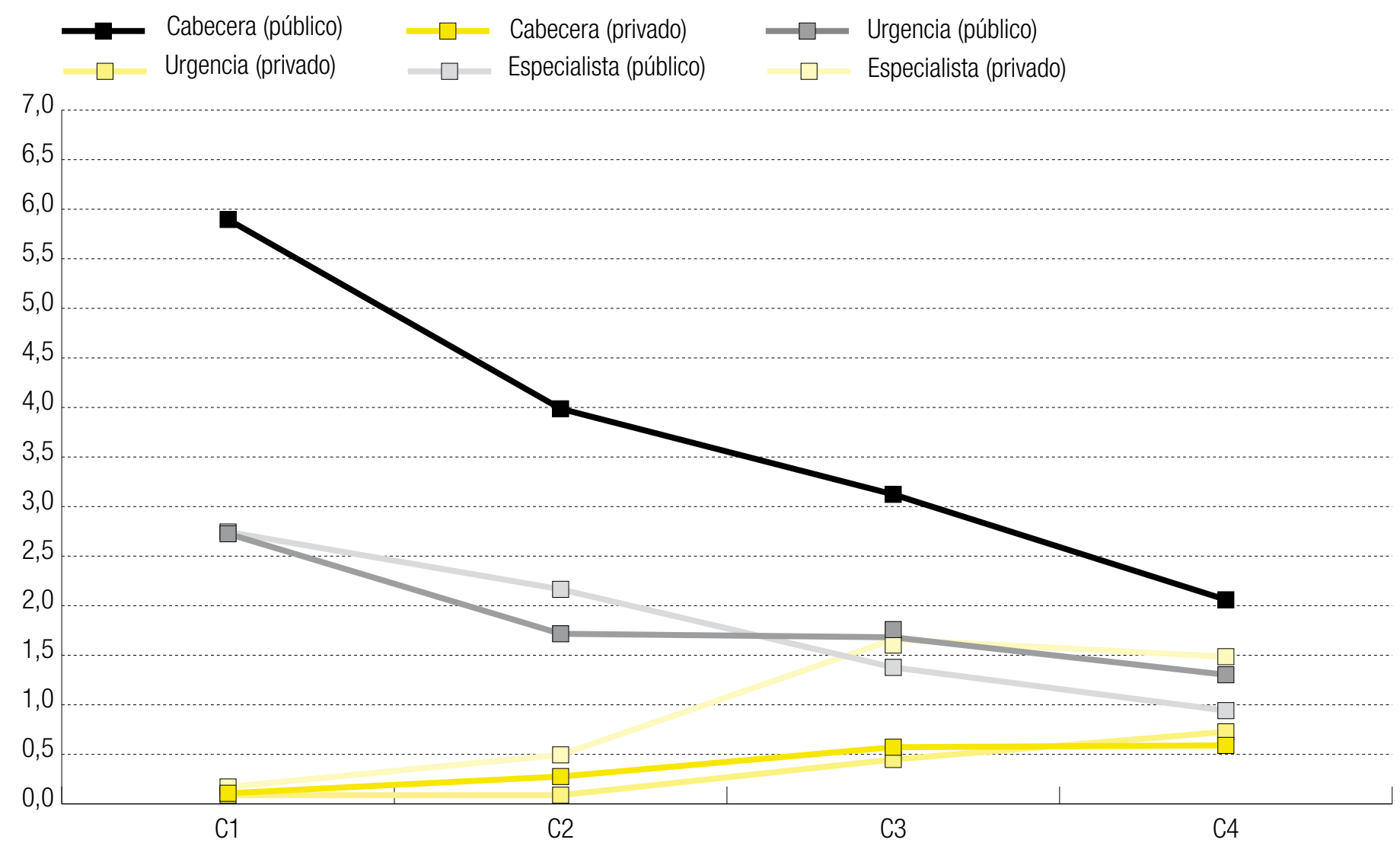

Fuente: elaboración propia a partir de los datos de la encuesta del Proyecto IMPACT-A.

Si bien, en términos generales, no se detectan diferencias estadísticamente significativas en función de los ingresos (medidos por cuartiles $-\mathrm{C} 1, \mathrm{C} 2$, C3 y C4-) en el uso de los servicios de salud, sí se aprecian claramente cuando comparamos el uso de los servicios públicos y privados. La figura 6 compara el número medio de asistencias a servicios de atención primaria, urgencias y especializados de carácter público y privado. En este caso, se observa que a medida que aumenta el nivel de ingresos la opción de recurrir a servicios privados de salud aumenta progresivamente. A partir de este gráfico se puede apreciar que la opción mayoritaria entre los andaluces es la pública, lo cual tiene sentido teniendo en cuenta que dentro del sistema sanitario en Andalucía el sector público es el predominante. No obstante, se pone de manifiesto un importante gradiente de salud en función de los ingresos. Así, por término medio, las personas con menores ingresos han acudido unas seis veces al médico de cabecera (público) frente a aquellos con ingresos más elevados que solo acuden dos. Mientras, en el caso del uso de servicios especializados de carácter privado dicha tendencia se invierte, siendo los individuos con más ingresos los que en mayor medida optan por esta vía. Estos datos ponen de manifiesto el problema al que muchas familias andaluzas pueden haberse visto sometidas a consecuencia de los problemas económicos experimentados durante el periodo de recesión económica. 
Tabla 2A. Dimensiones de salud de la escala SF12

\begin{tabular}{|c|c|c|c|c|c|c|}
\hline & $\begin{array}{l}\text { Estado de salud } \\
\text { general (personas } \\
\text { con mala salud) }\end{array}$ & $\begin{array}{l}\text { Limitaciones } \\
\text { físicas al realizar } \\
\text { esfuerzos }\end{array}$ & $\begin{array}{l}\text { Presenta } \\
\text { limitaciones para } \\
\text { subir escaleras }\end{array}$ & $\begin{array}{l}\text { Hizo menos de } \\
\text { lo que quería por } \\
\text { problemas físicos }\end{array}$ & $\begin{array}{l}\text { Tuvo que dejar de } \\
\text { hacer algunas de } \\
\text { sus actividades } \\
\text { cotidianas }\end{array}$ & $\begin{array}{l}\text { Hizo menos de } \\
\text { lo que quería por } \\
\text { algún problema } \\
\text { emocional }\end{array}$ \\
\hline Hombre & $15,7 \%$ & $15,3 \%$ & $14,3 \%$ & $18,7 \%$ & $17,9 \%$ & $18,7 \%$ \\
\hline Mujer & $23,4 \%$ & $31,1 \%$ & $26,0 \%$ & $28,9 \%$ & $26,6 \%$ & $28,1 \%$ \\
\hline Sig. & 0,000 & 0,000 & 0,000 & 0,000 & 0,000 & 0,000 \\
\hline $18-29$ & $5,1 \%$ & $8,3 \%$ & $3,9 \%$ & $18,0 \%$ & $14,1 \%$ & $19,2 \%$ \\
\hline $30-39$ & $7,0 \%$ & $9,9 \%$ & $8,1 \%$ & $15,1 \%$ & $14,4 \%$ & $18,3 \%$ \\
\hline $40-49$ & $18,3 \%$ & $19,1 \%$ & $15,9 \%$ & $18,3 \%$ & $18,7 \%$ & $24,2 \%$ \\
\hline $50-59$ & $24,9 \%$ & $23,3 \%$ & $21,2 \%$ & $26,4 \%$ & $24,4 \%$ & $24,4 \%$ \\
\hline $60-69$ & $31,2 \%$ & $34,8 \%$ & $30,4 \%$ & $30,4 \%$ & $28,3 \%$ & $22,5 \%$ \\
\hline $70-79$ & $33,9 \%$ & $46,6 \%$ & $39,8 \%$ & $33,1 \%$ & $30,5 \%$ & $28,8 \%$ \\
\hline 80 o más & $50,9 \%$ & $72,9 \%$ & $74,6 \%$ & $64,4 \%$ & $61,0 \%$ & $45,8 \%$ \\
\hline Sig. & 0,000 & 0,000 & 0,000 & 0,000 & 0,000 & 0,000 \\
\hline C1 & $33,2 \%$ & $38,5 \%$ & $33,9 \%$ & $35,9 \%$ & $34,5 \%$ & $34,2 \%$ \\
\hline C2 & $23,3 \%$ & $23,7 \%$ & $23,3 \%$ & $25,4 \%$ & $24,1 \%$ & $26,3 \%$ \\
\hline C3 & $11,7 \%$ & $15,6 \%$ & $11,2 \%$ & $16,9 \%$ & $14,3 \%$ & $18,7 \%$ \\
\hline C4 & $8,1 \%$ & $12,2 \%$ & $8,1 \%$ & $13,5 \%$ & $15,5 \%$ & $10,8 \%$ \\
\hline Sig. & 0,000 & 0,000 & 0,000 & 0,000 & 0,000 & 0,000 \\
\hline Primaria & $47,8 \%$ & $59,8 \%$ & $53,3 \%$ & $52,2 \%$ & $53,3 \%$ & $43,5 \%$ \\
\hline Secundaria & $29,3 \%$ & $29,9 \%$ & $27,2 \%$ & $26,3 \%$ & $25,7 \%$ & $24,2 \%$ \\
\hline Universidad & $15,4 \%$ & $20,0 \%$ & $17,6 \%$ & $21,9 \%$ & $18,7 \%$ & $24,1 \%$ \\
\hline Postgrado & $8,8 \%$ & $12,8 \%$ & $9,5 \%$ & $17,5 \%$ & $16,0 \%$ & $18,0 \%$ \\
\hline Sig. & 0,000 & 0,000 & 0,000 & 0,000 & 0,000 & 0,000 \\
\hline Almería & $26,7 \%$ & $25,3 \%$ & $20,0 \%$ & $26,7 \%$ & $24,7 \%$ & $24,0 \%$ \\
\hline Cádiz & $14,7 \%$ & $27,3 \%$ & $18,7 \%$ & $29,3 \%$ & $23,3 \%$ & $31,3 \%$ \\
\hline Córdoba & $16,7 \%$ & $24,0 \%$ & $18,0 \%$ & $18,7 \%$ & $16,7 \%$ & $24,0 \%$ \\
\hline Granada & $19,3 \%$ & $18,0 \%$ & $20,0 \%$ & $20,7 \%$ & $19,3 \%$ & $20,0 \%$ \\
\hline Huelva & $18,0 \%$ & $24,0 \%$ & $20,7 \%$ & $23,3 \%$ & $22,0 \%$ & $15,3 \%$ \\
\hline Jaén & $24,7 \%$ & $20,7 \%$ & $22,7 \%$ & $22,0 \%$ & $23,3 \%$ & $27,3 \%$ \\
\hline Málaga & $18,7 \%$ & $22,0 \%$ & $18,7 \%$ & $24,0 \%$ & $23,3 \%$ & $24,0 \%$ \\
\hline Sevilla & $18,0 \%$ & $25,3 \%$ & $23,3 \%$ & $26,7 \%$ & $26,0 \%$ & $22,0 \%$ \\
\hline Sig. & 0,142 & 0,633 & 0,935 & 0,414 & 0,604 & 0,068 \\
\hline
\end{tabular}

Fuente: elaboración propia a partir de los datos de la encuesta del Proyecto IMPACT-A. 


\subsection{La salud de la población andaluza: resultados de la escala SF12}

En este apartado se describen los resultados para los diferentes indicadores que componen la escala SF12. Este instrumento de medición de la salud general se compone de un total de 12 ítems que hacen referencia tanto a la salud física como la mental. Estos son los siguientes: (1) estado de salud general; (2) presentar limitaciones físicas al realizar esfuerzos; (3) presentar limitaciones para subir escaleras; (4) hizo menos de lo que quería por problemas físicos; (5) tuvo que dejar de hacer algunas de sus actividades cotidianas; (6) hizo menos de lo que quería por algún problema emocional; (7) no hizo sus tareas como de costumbre por problema emocional; (8) el dolor le ha dificultado su trabajo habitual; (9) ¿se sintió calmado?; (10) ¿tuvo mucha energía?; (11) ¿se sintió desanimado o triste?, y (12) la salud le dificulta sus actividades sociales.

Cuando nos centramos en la autopercepción de salud podemos apreciar que las mujeres reportan una peor salud (23,4 \%) que los hombres (15,7 \%). Así, casi una de cada cuatro andaluzas define su salud como mala, mientras que los hombres no llegan a ser dos de cada diez. Igualmente, estas diferencias estadísticamente significativas en relación al sexo de la persona entrevistada, se reproducen en el resto de los indicadores de la escala. Las mujeres andaluzas reportan mayores limitaciones físicas, así como problemas para llevar a cabo las actividades cotidianas. Por ejemplo, el 31,1\% de las andaluzas admiten tener limitaciones para realizar esfuerzos frente al 15,3\% de los hombres, y el 26,6\% presenta limitaciones a la hora de realizar las actividades cotidianas frente al 17,9\% de los hombres. Las mujeres experimentan dolores en mayor medida que los hombres, y también presentan un estado psicológico y emocional más deteriorado que estos. En efecto, un 10,6 \% afirma sentirse triste siempre o casi siempre frente al 6,8 \% de los hombres, un dato que apunta a que las andaluzas presentan peor salud mental que los hombres. Por otra parte, la edad es un factor fundamental a la hora de explicar las diferencias de salud. Si bien, es esperable que sean los grupos de mayor edad aquellos que presentan una peor salud física, llama la atención el hecho de que en términos de bienestar emocional, una de cada cuatro personas de 80 años o más se sientan tristes siempre o casi siempre (27,1\%), así como el hecho de que una de cada cinco presentan problemas para llevar a cabo actividades de carácter social (20,3\%). En definitiva, unos datos que ponen de manifiesto la peor calidad de vida de estos grupos altamente vulnerables.

\section{Tabla 3. Dificultades de salud asociadas a la crisis}

\begin{tabular}{|c|c|c|c|}
\hline & $\begin{array}{l}\text { Considera que } \\
\text { la crisis le ha } \\
\text { afectado a su } \\
\text { salud }\end{array}$ & $\begin{array}{l}\text { Ha tenido } \\
\text { dificultades } \\
\text { económicas } \\
\text { para acceder a } \\
\text { tratamientos }\end{array}$ & $\begin{array}{l}\text { Necesidades } \\
\text { médicas no } \\
\text { cumplidas }\end{array}$ \\
\hline Hombre & $14,7 \%$ & $8,7 \%$ & $8,2 \%$ \\
\hline Mujer & $20,0 \%$ & $16,7 \%$ & $13,2 \%$ \\
\hline Sig. & 0,015 & 0,000 & 0,000 \\
\hline $18-29$ & $17,4 \%$ & $16,7 \%$ & $9,0 \%$ \\
\hline $30-39$ & $19,0 \%$ & $10,9 \%$ & $12,3 \%$ \\
\hline 40-49 & $19,7 \%$ & $15,1 \%$ & $13,6 \%$ \\
\hline $50-59$ & $19,7 \%$ & $14,1 \%$ & $9,9 \%$ \\
\hline $60-69$ & $11,0 \%$ & $12,3 \%$ & $11,6 \%$ \\
\hline 70-79 & $13,6 \%$ & $6,8 \%$ & $6,8 \%$ \\
\hline 80 o más & $15,5 \%$ & $10,2 \%$ & $5,1 \%$ \\
\hline Sig. & 0,283 & 0,192 & 0,282 \\
\hline $\mathrm{C} 1$ & $31,4 \%$ & $25,0 \%$ & $14,8 \%$ \\
\hline C2 & $22,0 \%$ & $12,1 \%$ & $11,6 \%$ \\
\hline C3 & $10,9 \%$ & $8,8 \%$ & $10,4 \%$ \\
\hline C4 & $5,4 \%$ & 6.8 & 7.5 \\
\hline Sig. & 0.000 & 0.000 & 0.050 \\
\hline Primaria & 16,5 & 12,0 & 9,8 \\
\hline Secundaria & 19,6 & $13,7 \%$ & $8,7 \%$ \\
\hline Universidad & $18,4 \%$ & $15,7 \%$ & $13,8 \%$ \\
\hline Postgrado & $15,1 \%$ & $9,5 \%$ & $10,0 \%$ \\
\hline Sig. & 0,410 & 0,075 & 0,144 \\
\hline Almería & $22,7 \%$ & $11,4 \%$ & $9,3 \%$ \\
\hline Cádiz & $23,3 \%$ & $12,0 \%$ & $8,7 \%$ \\
\hline Córdoba & $16,0 \%$ & $18,7 \%$ & $12,0 \%$ \\
\hline Granada & $12,1 \%$ & $11,3 \%$ & $8,7 \%$ \\
\hline Huelva & $12,0 \%$ & $11,3 \%$ & $10,0 \%$ \\
\hline Jaén & $15,4 \%$ & $7,3 \%$ & $10,1 \%$ \\
\hline Málaga & $20,3 \%$ & $17,3 \%$ & $16,0 \%$ \\
\hline Sevilla & $17,6 \%$ & $12,7 \%$ & $11,3 \%$ \\
\hline Sig. & 0,050 & 0,089 & 0,495 \\
\hline
\end{tabular}




\section{Tabla 2B. Dimensiones de salud de la escala SF12}

\begin{tabular}{|c|c|c|c|c|c|c|}
\hline & $\begin{array}{l}\text { No hizo sus tareas } \\
\text { como de costumbre } \\
\text { por problema } \\
\text { emocional }\end{array}$ & $\begin{array}{l}\text { El dolor le ha } \\
\text { dificultado su } \\
\text { trabajo habitual }\end{array}$ & $\begin{array}{l}\text { Se sintió calmado? } \\
\text { (siempre o casi } \\
\text { siempre) }\end{array}$ & $\begin{array}{l}\text { Tuvo mucha } \\
\text { energía? (siempre o } \\
\text { casi siempre) }\end{array}$ & $\begin{array}{l}\text { Se sintió } \\
\text { desanimado o } \\
\text { triste? (siempre o } \\
\text { casi siempre) }\end{array}$ & $\begin{array}{l}\text { La salud le dificulta } \\
\text { sus actividades } \\
\text { sociales (siempre o } \\
\text { casi siempre) }\end{array}$ \\
\hline Hombre & $13,6 \%$ & $27,9 \%$ & $64,1 \%$ & $56,29 \%$ & $6,8 \%$ & $6,1 \%$ \\
\hline Mujer & $21,7 \%$ & $42,8 \%$ & $47,9 \%$ & $40,36 \%$ & $10,6 \%$ & $11,0 \%$ \\
\hline Sig. & 0,000 & 0,000 & 0,000 & 0,000 & 0,000 & 0,000 \\
\hline $18-29$ & $12,8 \%$ & $27,6 \%$ & $49,4 \%$ & $51,9 \%$ & $2,6 \%$ & $3,9 \%$ \\
\hline 30-39 & $13,4 \%$ & $24,3 \%$ & $55,6 \%$ & $54,6 \%$ & $5,6 \%$ & $3,5 \%$ \\
\hline $40-49$ & $16,7 \%$ & $36,5 \%$ & $48,0 \%$ & $42,9 \%$ & $6,8 \%$ & $8,7 \%$ \\
\hline $50-59$ & $18,1 \%$ & $35,8 \%$ & $58,6 \%$ & $49,2 \%$ & $7,3 \%$ & $7,8 \%$ \\
\hline $60-69$ & $16,7 \%$ & $44,2 \%$ & $71,7 \%$ & $54,4 \%$ & $10,1 \%$ & $12,3 \%$ \\
\hline 70-79 & $25,4 \%$ & $44,1 \%$ & $61,0 \%$ & $39,8 \%$ & $20,3 \%$ & $17,8 \%$ \\
\hline 80 o más & $42,4 \%$ & $67,8 \%$ & $50,9 \%$ & $28,8 \%$ & $27,1 \%$ & $20,3 \%$ \\
\hline Sig. & 0,000 & 0,000 & 0,000 & 0,000 & 0,000 & 0,000 \\
\hline $\mathrm{C} 1$ & $28,0 \%$ & $52,3 \%$ & $46,1 \%$ & $38,5 \%$ & $18,8 \%$ & $20,4 \%$ \\
\hline C2 & $19,8 \%$ & $36,6 \%$ & $57,3 \%$ & $45,7 \%$ & $9,5 \%$ & $9,1 \%$ \\
\hline C3 & $13,3 \%$ & $28,1 \%$ & $59,0 \%$ & $53,0 \%$ & $3,9 \%$ & $2,9 \%$ \\
\hline C4 & $8,8 \%$ & $24,3 \%$ & $62,2 \%$ & $56,1 \%$ & $1,4 \%$ & $3,4 \%$ \\
\hline Sig. & 0,000 & 0,000 & 0,000 & 0,000 & 0,000 & 0,000 \\
\hline Primaria & $42,4 \%$ & $65,2 \%$ & $46,7 \%$ & $26,1 \%$ & $31,5 \%$ & $26,1 \%$ \\
\hline Secundaria & $18,8 \%$ & $41,8 \%$ & $57,0 \%$ & $46,0 \%$ & $9,6 \%$ & $11,0 \%$ \\
\hline Universidad & $16,0 \%$ & $33,8 \%$ & $53,8 \%$ & $51,4 \%$ & $7,8 \%$ & $6,2 \%$ \\
\hline Postgrado & $13,0 \%$ & $25,0 \%$ & $59,0 \%$ & $52,3 \%$ & $3,8 \%$ & $4,8 \%$ \\
\hline Sig. & 0,000 & 0,000 & 0,000 & 0,000 & 0,000 & 0,000 \\
\hline Almería & $20,0 \%$ & $413 \%$ & $58,7 \%$ & $49,3 \%$ & $8,7 \%$ & $8,7 \%$ \\
\hline Cádiz & $22,0 \%$ & $35,3 \%$ & $59,3 \%$ & $48,0 \%$ & $6,7 \%$ & $8,7 \%$ \\
\hline Córdoba & $19,3 \%$ & $31,3 \%$ & $50,0 \%$ & $48,0 \%$ & $9,3 \%$ & $7,3 \%$ \\
\hline Granada & $18,0 \%$ & $28,7 \%$ & $56,7 \%$ & $50,0 \%$ & $8,0 \%$ & $6,7 \%$ \\
\hline Huelva & $12,0 \%$ & $32,7 \%$ & $58,0 \%$ & $55,3 \%$ & $12,7 \%$ & $7,3 \%$ \\
\hline Jaén & $18,0 \%$ & $32,0 \%$ & $54,7 \%$ & $43,3 \%$ & $7,3 \%$ & $12,7 \%$ \\
\hline Málaga & $16,0 \%$ & $36,7 \%$ & $59,3 \%$ & $50,7 \%$ & $8,7 \%$ & $8,0 \%$ \\
\hline Sevilla & $16,7 \%$ & $46,0 \%$ & $50,0 \%$ & $40,7 \%$ & $8,7 \%$ & $9,3 \%$ \\
\hline Sig. & 0,485 & 0,035 & 0,480 & 0,288 & 0,751 & 0,793 \\
\hline
\end{tabular}

Fuente: elaboración propia a partir de los datos de la encuesta del Proyecto IMPACT-A.

En los estudios previos, se pone de manifiesto la relación positiva entre el estatus socioeconómico y la salud a través de las diferencias en función del nivel de ingresos y educativo (Álvarez-Gálvez et al. 2013, 2014; Eikemo et al., 2008). Uno de cada tres andaluces en el tramo más bajo de ingresos reporta mala salud (33,2 \%), mientras que no llega a uno de cada diez entre aquellos que presentan los ingresos más elevados (8,1\%). La distribución de ingresos describe un importante gradiente de salud física y mental entre aquellos que tienen más y aquellos que tienen menos. En el caso de la salud física el grupo 
Se observan mayores diferencias entre hombres $(1,1 \%)$ y mujeres $(13,8 \%)$ en términos de los efectos de la crisis sobre la salud, un dato que vuelve a poner de manifiesto la brecha de género en el reparto de las tareas del hogar y el trabajo remunerado

con mayores ingresos suele triplicar la puntuación de salud de aquellos que se sitúan en el cuartil de ingresos más bajo, aunque estas diferencias aumentan enormemente cuando nos referimos a la experimentación de sentimientos de tristeza habituales o a las dificultades para llevar a cabo actividades sociales. Así, mientras que el 18,8 \% de las personas con menos ingresos admiten sentirse tristes siempre o casi siempre, solo un 1,4 \% de aquellos con ingresos elevados experimentan sentimientos de tristeza. En el caso de tener problemas con las relaciones sociales, estas diferencias se mantienen un rango de $20,4 \%$ a 3,4 \%. En función del nivel educativo la tendencia es similar, esto es, a mayor nivel educativo mejor salud física y mental. No obstante, debemos considerar el efecto generacional en estas diferencias.

En función de la provincia, no se detectan diferencias estadísticamente significativas. Aunque se aprecia una cierta variabilidad en los porcentajes, no se observa una tendencia clara que pudiera apuntar a la existencia de desigualdades de salud basándose en los diferentes territorios de la comunidad andaluza.

\subsection{Dificultades sanitarias asociadas a la crisis económica}

Cuando nos centramos en las dificultades sanitarias asociadas al momento de crisis económica, podemos apreciar que las mujeres se han visto más afectadas que los hombres en términos de salud, con un porcentaje del $20 \%$ de mujeres frente al 14,7 \% de los hombres (tabla 3). Del mismo modo, también han experimentado mayores dificultades para acceder a tratamientos de salud $y$, en mayor medida que los hombres, consideran que sus necesidades de salud no se encuentran totalmente satisfechas. La situación de necesidad o dificultades económicas no tiene una relación estadísticamente significativa con la edad, aunque resulta llamativo el hecho de que en general los grupos más jóvenes presenten porcentajes superiores a los de los grupos de mayor edad, lo cual puede ser un signo real de los problemas que actualmente experimenta la juventud andaluza con las elevadas tasas de desempleo juvenil y consiguiente acceso a puestos de calidad dentro del mercado de trabajo.

En función de los ingresos, se puede apreciar que son los grupos situados en el cuarto cuartil de ingresos los que, lógicamente, han experimentado menos problemas de salud o en el acceso a tratamientos y servicios de salud. En el extremo opuesto, entre los grupos con menores ingresos, un 31,4 \% afirma haber tenido dificultades de salud como consecuencia de la crisis económica, un $25 \%$ ha tenido dificultades en el acceso a tratamientos y un $15,8 \%$ indica que sus necesidades de salud no se encuentran totalmente cubiertas. Los datos no presentan una asociación negativa con la experimentación de estas dificultades, aunque de nuevo resulta reseñable el hecho de que los porcentajes resulten superiores entre el grupo de los universitarios. Finalmente, en función de la provincia de residencia, encontramos diferencias estadísticamente significativas entre aquellos que consideran que su salud se ha visto afectada por la crisis. En este caso, la provincia que parece haberse visto más afectada es Cádiz con un 23,3 \%, mientras que en el extremo opuesto Huelva presenta un $12 \%$.

En función de la situación laboral de la persona entrevistada, podemos apreciar que el mayor impacto de la crisis ha recaído tanto sobre las personas que actualmente trabajan como en aquellos que se encontraban en situación desempleo, sobre todo en hombres (figura 7). En el caso de las mujeres, las peor paradas han sido aquellas que se encuentran jubiladas o son pensionistas, así como las que realizan trabajo doméstico no remunerado. Es en esta última categoría donde se observan mayores diferencias entre hombres $(1,1 \%)$ y mujeres $(13,8 \%)$ en términos de los efectos de la crisis sobre la salud, un dato que vuelve a poner de manifiesto la brecha de género en el reparto de las tareas del hogar y el trabajo remunerado.

Ahora bien, cuando hablamos de necesidades de salud no cumplidas ¿a qué nos referimos exactamente? En la figura 8 se muestran las necesidades que en mayor medida fueron mencionadas por los informantes. Como se puede observar, los problemas dentales ocupan la primera posición en el ranking de necesidades con un 62,7 \%, seguidos por los tratamientos de carácter fisioterapéutico y operaciones relacionadas con el aparato locomotor con un $13,3 \%$, y el acceso a medicamentos en general con un 7,6\%. No obstante, se menciona la necesidad de visitar al endocrino en busca de ayuda para el seguimiento de dietas y hábitos saludables, al ginecólogo, al especialista en salud mental, óptica, dermatología, problemas crónicos como las migrañas, así como la ausencia de alternativas médicas en el caso de enfermedades raras. 
Figura 7. Porcentaje de andaluces que afirman que su estado de salud se ha visto afectado por su situación económica, según la situación laboral

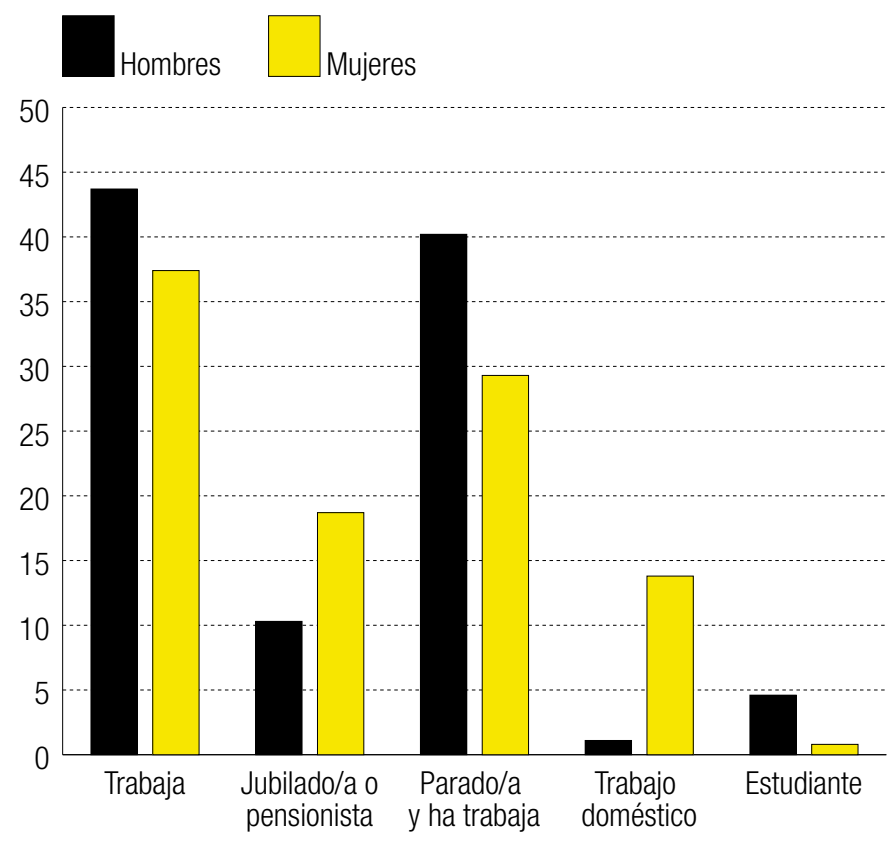

Fuente: elaboración propia a partir de los datos de la encuesta del Proyecto IMPACT-A.

\subsection{Percepción de discriminación y salud en Andalucía}

Seguidamente, se ha encontrado que un 8,3\% de los andaluces se siente parte de un grupo discriminado en este país $(n=100)$, sin encontrarse diferencias significativas de género. En la figura 9 podemos observar que los porcentajes más elevados (ligeramente por encima del $10 \%$ ) se encuentran en los hombres de entre 35 y 64 años. La mayor diferencia de género en esta variable de estudio se encuentra en los andaluces de mayor edad, donde más del doble de varones que de mujeres se sienten discriminados. Entre los andaluces encuestados que se sienten discriminados, se han encontrado diversos motivos asociados al color o raza, nacionalidad, religión, edad, género, orientación sexual, discapacidad, nivel educativo y clase social.

De las personas que reportaban sentirse discriminadas, un 9,1\% indicaban razones de género y discapacidad. Curiosamente, el $65 \%$ de los entrevistados hacía referencia a «otras» causas de discriminación (figura 10), algo atípico teniendo en cuenta que — por término medio - en los países de la Unión Europea las personas que reportan otras causas «no habituales» dentro de la investigación académica rondan el 1-5 \% según los datos de la Encuesta Social Europea (Álvarez-Gálvez y Salvador-Carulla, 2013; ÁlvarezGálvez, 2016). Al analizar los resultados de la pregunta abierta que examinaba esas otras causas de discriminación, llama la atención el hecho de

\section{Figura 8. Necesidades de salud no cumplidas entre la población andaluza}

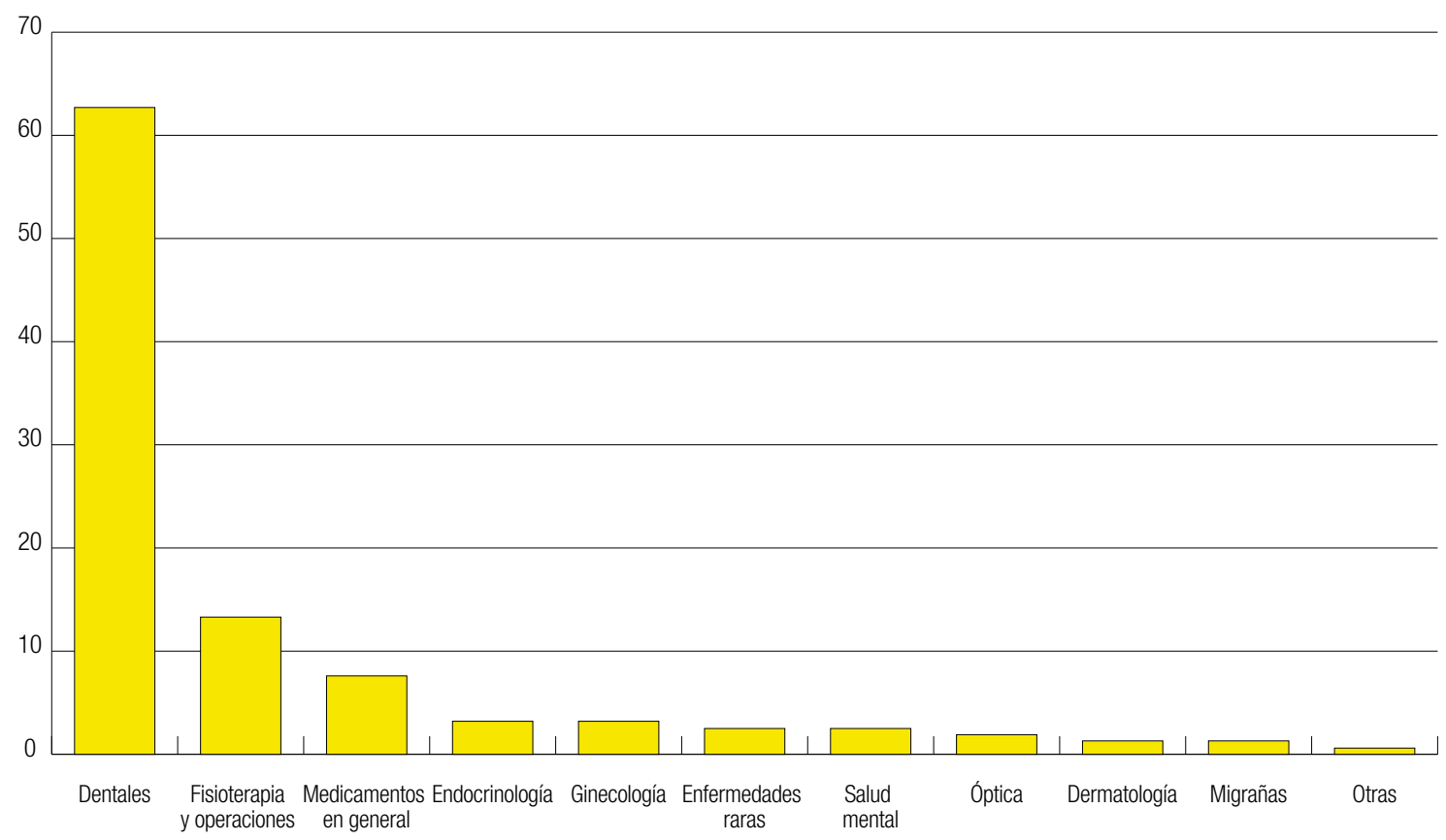

Fuente: elaboración propia a partir de los datos de la encuesta del Proyecto IMPACT-A. 
Figura 9. Descripción como miembro de un grupo
discriminado en este país, por género y edad

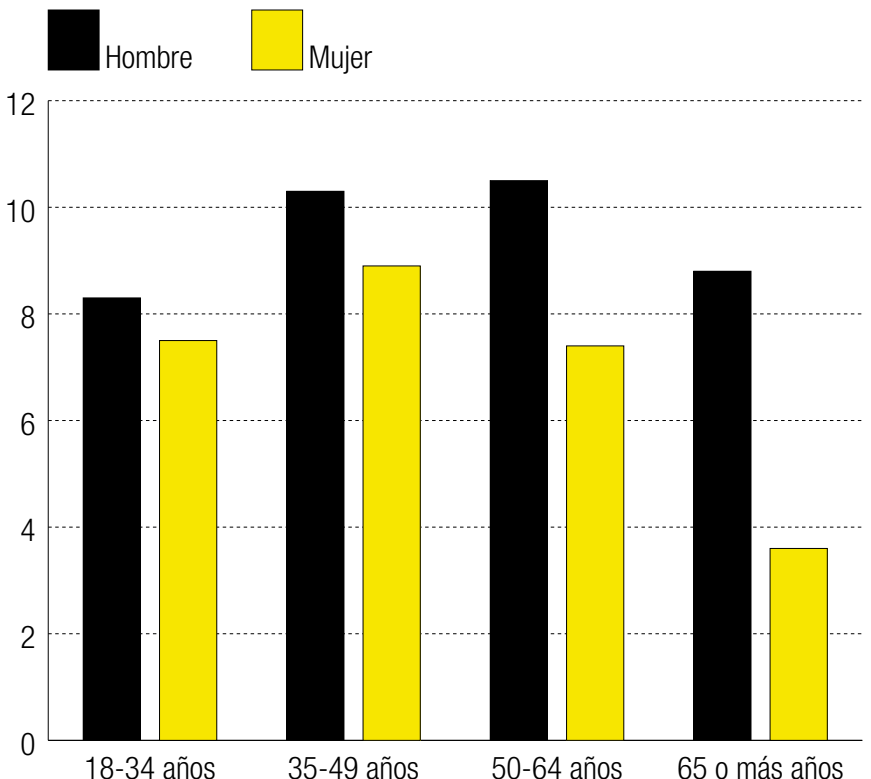

Fuente: elaboración propia a partir de los datos de la encuesta del Proyecto IMPACT-A. que todas las respuestas hacían referencia a discriminación laboral. Las principales causas de discriminación laboral percibida eran la siguientes: los problemas para encontrar trabajo, el desempleo de larga duración, las dificultades para encontrar trabajo de mayores y jóvenes, la discriminación laboral a mujeres en edad fértil, la infravaloración de las tareas realizadas en el trabajo, la realización de trabajos precarios, 0 incluso el hecho de ser trabajadores autónomos. Unos datos que apuntan a la precaria situación laboral y económica de muchos andaluces y andaluzas que no tienen acceso al mercado de trabajo o tienen una situación de riesgo dentro del mismo.

Al comparar la discriminación laboral con respecto a otras causas comunes de discriminación, raza, género, religión, nacionalidad, etc., se evidencia el mismo efecto negativo para la salud. En otras palabras, podemos decir que sentirse discriminado por temas laborales resulta igualmente nocivo para nuestra salud que sentirse discriminado por motivos sociodemográficos, étnicos y/o culturales. Por otro lado, se pone de manifiesto un mayor uso de los servicios de atención primaria entre aquellos que se sienten laboral o económicamente discriminados (figura 11), un dato que demuestra la mayor prevalencia de problemas de salud entre los grupos que se enfrentan al desempleo y/o al trabajo precario. Además, esta asociación entre la discriminación laboral y los peores resultados de salud resulta fundamental para explicar cómo la crisis económica, la falta de ayudas y el aumento del

\section{Figura 10. Causas de discriminación}

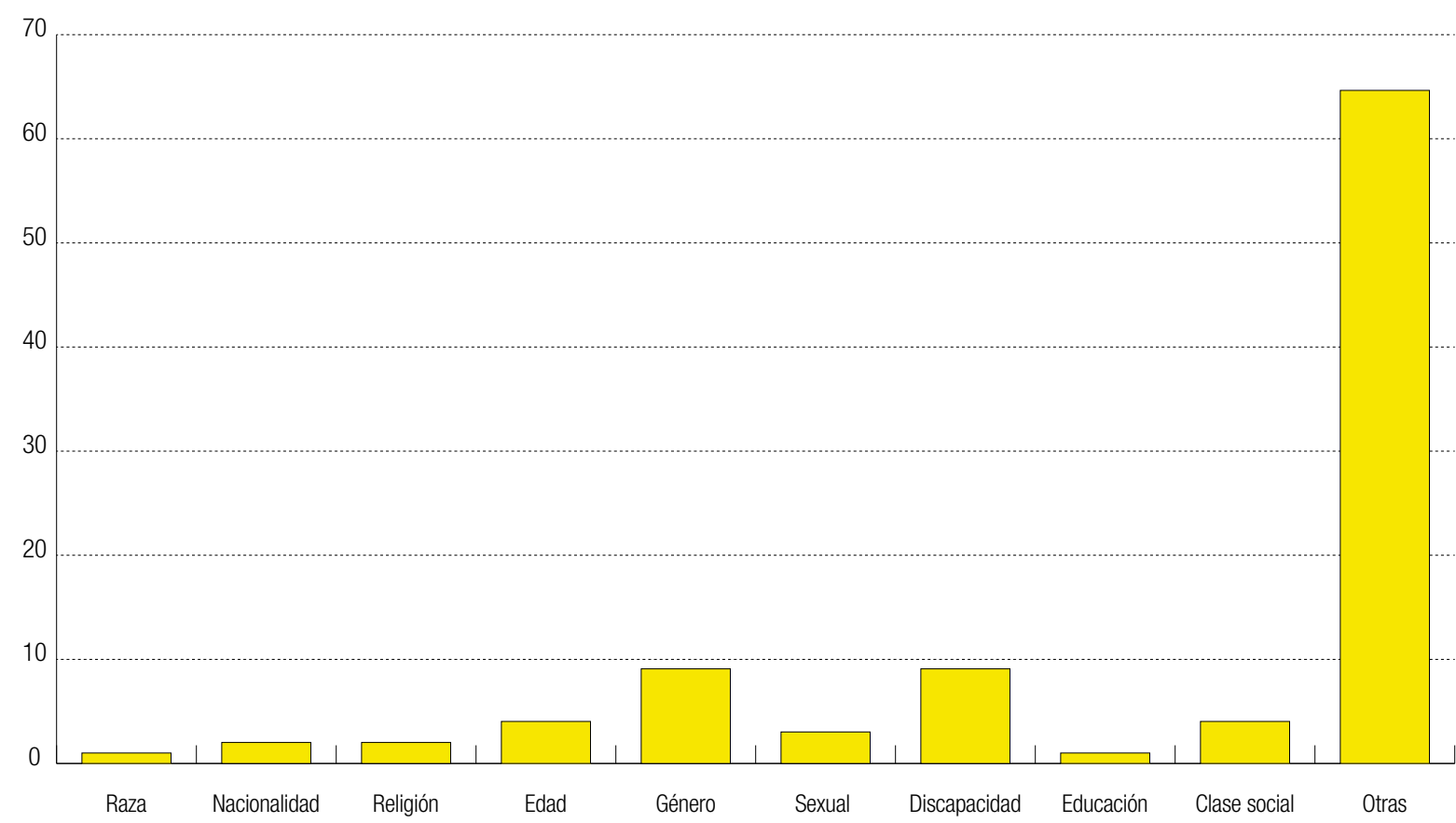

Fuente: elaboración propia a partir de los datos de la encuesta del Proyecto IMPACT-A. 


\section{Figura 11. Comparación del uso de servicios de salud de atención primaria en función de la causa de discriminación}

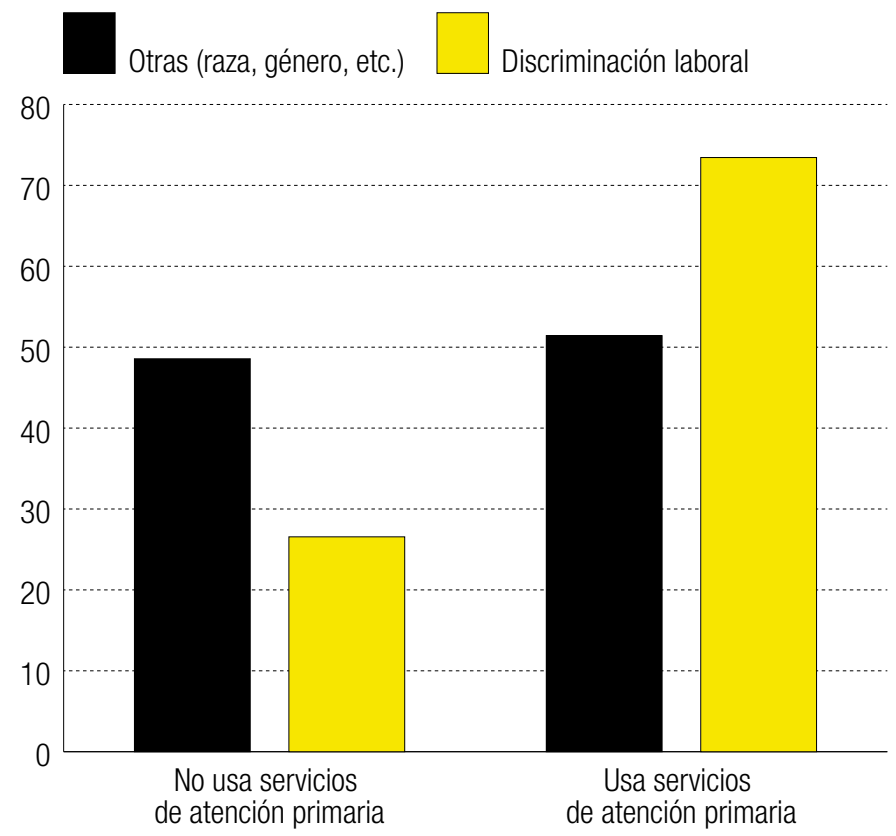

Fuente: elaboración propia a partir de los datos de la encuesta del Proyecto IMPACT-A.

desempleo han podido incrementar los problemas de autoestima, insomnio, estrés y trastornos depresivos de los grupos que más se han visto afectados por los vaivenes económicos de los últimos años.

En definitiva, estos resultados apuntan a la precaria situación laboral y económica de muchos andaluces y andaluzas que no tienen acceso al mercado de trabajo o tienen una situación de riesgo dentro del mismo. Una situación de riesgo y vulnerabilidad social que puede traducirse en futuras desigualdades de salud y, consecuentemente, en mayores problemas socioeconómicos a través de la progresiva acumulación de desigualdades a lo largo del ciclo vital.

\section{Sentirse discriminado por temas}

laborales resulta igual de nocivo para nuestra salud que sentirse discriminado por motivos sociodemográficos, étnicos y/o culturales

\section{Determinantes sociales de la salud en Andalucía: análisis por provincias}

Los datos de la encuesta ponen de manifiesto la existencia de diferencias de salud entre los diferentes grupos analizados. En concreto, se detecta la persistencia de desigualdades sanitarias y de calidad de vida de los andaluces asociadas a determinantes sociales de salud como son el género, la percepción de discriminación, la distribución de ingresos, la educación, la situación laboral y los estilos de vida (practicar deportes, llevar una dieta sana, etc.). En esta sección se llevan a cabo diferentes modelos de regresión para comparar el impacto de los factores analizados en los apartados previos (concretamente aquellos que resultaron estadísticamente significativos) sobre el estado general de salud, medido a través del índice SF12. Esta escala de salud oscila entre los valores 0 y 100, donde 0 sería el peor estado de salud posible frente a 100 que sería el mejor.

Los predictores que se han incluido en los modelos han sido los siguientes: (1) los ingresos mensuales; (2) el nivel de estudios; (3) si la persona considera que su salud se ha visto afectada por la crisis; (4) si la persona ha pasado dificultades económicas para acceder a tratamientos médicos durante la crisis; (5) si la persona considera que tiene necesidades sanitarias no-cumplidas; (6) la percepción de felicidad; (7) sentirse discriminado; (8) la frecuencia de consumo de fruta; (9) la frecuencia de consumo de verdura; (10) frecuencia con la que practica alguna actividad física; (11) el índice de masa corporal (IMC); (12) si la persona fuma (o ha fumado); (13) la frecuencia de consumo de alcohol; (14) haber asistido a una consulta de atención primaria (últimos 12 meses); (15) haber asistido a urgencias (últimos 12 meses); (16) haber asistido a una consulta especializada (últimos 12 meses); (17) edad; (18) sexo (ser mujer); y (19) el tamaño del hábitat.

A partir de la tabla 4, se observa que para el conjunto de Andalucía, el determinante socioeconómico más relevante es la educación, el cual presenta una relación positiva con la salud, especialmente en las provincias de Jaén y Almería. Esta asociación positiva entre la educación y la salud ya se ha puesto de manifiesto en estudios previos para el conjunto del país (Eike- 
mo et al., 2008; Álvarez-Gálvez et al., 2013). Sin embargo, los ingresos no presentan una relación estadísticamente significativa en la explicación de la salud general de la población andaluza, lo que se debe en gran medida a la existencia de un sistema público de salud, que reduce significativamente las desigualdades por ingresos, las cuales son más evidentes en otros países con sistemas de salud predominantemente privados. Sin embargo, en provincias como Almería o Granada, el factor económico sí resulta relevante para explicar las diferencias de salud.

En segundo lugar, nos encontramos con las tres variables (3-5) que hacen referencia al posible impacto de la crisis sobre la salud de los andaluces. Estos tres predictores muestran una relación negativa con la salud. Estos datos ponen de relieve que los andaluces y andaluzas que consideran que la crisis les ha afectado (Cádiz, Granada y Málaga), que han podido sufrir dificultades económicas para acceder a tratamientos (Córdoba, Granada y Sevilla) o que tienen algunas necesidades médicas no cumplidas, presentan una peor salud que el resto (Almería y Sevilla). Lógicamente algunas relaciones pierden significatividad estadística al desagregar la muestra total por provincias, sin embargo, la tendencia similar de algunos coeficientes apuntan hacia la validez global de los resultados. A pesar de las bondades de nuestro sistema público de salud de cara a la reducción de ciertas desigualdades asociadas a los problemas económicos, llama la atención el hecho de que un 13 \% de Ios andaluces afirmen que han tenido problemas para acceder a algún medicamento 0 tratamiento. Cuando indagamos en cuáles son esas necesidades no cumplidas, salen a la luz los problemas de las listas de espera, la falta de servicios y profesionales sanitarios, así como los problemas económicos para mantener una adecuada salud bucodental.

La felicidad se encuentra claramente relacionada con la salud, lo cual también es debido a la correlación existente entre la dimensión psicológica de la escala SF12 y la felicidad. El sentirse feliz aumenta nuestra salud en el conjunto de Andalucía, y en todas las provincias con la excepción de Sevilla, aunque el coeficiente positivo apunta en la misma tendencia que el resto de provincias. En este sentido, es muy probable que esta relación se vuelva estadísticamente significativa con muestras mayores. Así, este resultado apunta en la dirección de muchos estudios que indican que la felicidad puede mejorar la salud (Lawrence, Rogers y Wadsworth, 2015), aunque siempre tenemos que considerar la posibilidad de una relación inversa, esto es, cuando los problemas de salud pueden afectar a nuestra felicidad. Por otra parte, las personas que se sienten discriminadas en Andalucía, con independencia del motivo que sea, también ven reducida su salud, especialmente dentro de las provincias de Cádiz, Granada y Huelva. Por consiguiente, se puede apreciar que las desigualdades de salud asociadas a procesos de discriminación y exclusión social también se reproducen dentro de nuestra comunidad, un fenómeno que también se da en el resto del país y en Europa (Álvarez-Gálvez y Salvador-Carulla, 2013). Ahora bien, aunque no tenemos un tamaño de muestra adecuado para poder llevar a cabo un análisis pormenorizado de cada uno de los motivos de discriminación, el presente trabajo ha puesto de relieve que además de las razones de discriminación usualmente analizadas dentro del ámbito científico de los determinantes sociales de la salud (discriminación por sexo, edad, raza, etnia, nacionalidad, religión, y orientación sexual), existen otras formas de rechazo como la discriminación laboral, cuyos efectos sobre la salud resultan a día de hoy invisibles.

En relación a los estilos de vida, se puede observar que la realización de actividades físicas y una dieta equilibrada, basada en el consumo de productos saludables, pueden incrementar nuestra salud general. Para el conjunto de Andalucía se aprecia el hecho de que comer fruta está positivamente relacionado con nuestra salud, aunque esta relación no se encuentra con la ingesta de verdura, a excepción de las provincias de Granada, Jaén y Sevilla. Por el contrario, el índice de masa corporal (IMC) presenta una relación negativa, lo cual indica que la obesidad reduce nuestra salud general. A partir de los datos obtenidos no se aprecia una asociación estadísticamente significativa entre el consumo de tabaco y la salud general de la población andaluza. El efecto negativo de tabaco solo se presenta entre fumadores frecuentes 0 aquellos que lo han sido en el pasado. Llama la atención la relación positiva entre el consumo de alcohol y la percepción general de salud, lo cual puede estar relacionado con la relativa normalización de este hábito en nuestro país, una práctica que desempeña un papel canalizador de las relaciones sociales en diferentes grupos de edad. En cualquier caso, debemos ser prudentes a la hora de interpretar esta relación, puesto que, la percepción de salud general se caracteriza por componentes subjetivos (el dolor que una persona siente, su grado de tristeza, su nivel de incapacidad, etc.), por lo que es probable que el empleo de biomarcadores objetivos redundará en la relación opuesta. Así pues, en futuros estudios sería necesario profundizar en la posible sobreestimación de la salud de los grupos que consumen bebidas alcohólicas en mayor medida.

Las asistencias al médico/a de cabecera, a servicios de urgencias y a consultas especializadas, se introdujeron en el modelo a modo de control para introducir el efecto moderador de las posibles enfermedades o problemas de salud dentro del análisis. Como cabría esperar, la utilización de estos servicios de salud se encuentra negativamente relacionada con la salud, esto es, acuden más a estos servicios las personas que están enfermas. El sexo, la edad y el tamaño del hábitat también se añadieron a los modelos a modo de control. La edad presenta la relación negativa esperable y el sexo vuelve a poner de manifiesto el peor estado de salud de las mujeres andaluzas frente a los hombres. En función de hábitat no se detectaron diferencias estadísticamente significativas. 
Tabla 4. Determinantes sociales de la salud en Andalucía

\begin{tabular}{|c|c|c|c|c|c|c|c|c|c|}
\hline & Almería & Cádiz & Córdoba & Granada & Huelva & Jaén & Málaga & Sevilla & Andalucía \\
\hline $\begin{array}{l}\text { Ingresos } \\
\text { mensuales }\end{array}$ & $\begin{array}{l}1,673^{\star \star \star} \\
(1,048)\end{array}$ & $\begin{array}{l}0,048 \\
(0,959)\end{array}$ & $\begin{array}{l}-1,156 \\
(0,899)\end{array}$ & $\begin{array}{l}1,521^{*} \\
(0,885)\end{array}$ & $\begin{array}{l}-0,094 \\
(0,850)\end{array}$ & $\begin{array}{l}-0,932 \\
(0,966)\end{array}$ & $\begin{array}{l}-0,357 \\
(0,929)\end{array}$ & $\begin{array}{l}0,132 \\
(0,856)\end{array}$ & $\begin{array}{l}0,180 \\
(0,307)\end{array}$ \\
\hline $\begin{array}{l}\text { Nivel de } \\
\text { estudios }\end{array}$ & $\begin{array}{l}3,781^{\star *} \\
(1,715)\end{array}$ & $\begin{array}{l}1,335 \\
(1,593)\end{array}$ & $\begin{array}{l}0,237 \\
(1,787)\end{array}$ & $\begin{array}{l}-1,289 \\
(1,631)\end{array}$ & $\begin{array}{l}2,853 \\
(1,938)\end{array}$ & $\begin{array}{l}3,616^{\star \star} \\
(1,819)\end{array}$ & $\begin{array}{l}0,513 \\
(1,913)\end{array}$ & $\begin{array}{l}2,646 \\
(1,923)\end{array}$ & $\begin{array}{l}1,532^{\star \star \star} \\
(0,593)\end{array}$ \\
\hline $\begin{array}{l}\text { Le afectó la } \\
\text { crisis }\end{array}$ & $\begin{array}{l}-0,521 \\
(3,228)\end{array}$ & $\begin{array}{l}-7,872^{\star \star} \\
(3,783)\end{array}$ & $\begin{array}{l}-4,160 \\
(3,676)\end{array}$ & $\begin{array}{l}-6,973^{*} \\
(3,835)\end{array}$ & $\begin{array}{l}-4,300 \\
(3,739)\end{array}$ & $\begin{array}{l}-5,952 \\
(3,959)\end{array}$ & $\begin{array}{l}-5,982^{*} \\
(3,564)\end{array}$ & $\begin{array}{l}-2,974 \\
(4,107)\end{array}$ & $\begin{array}{l}-5,420^{\star \star *} \\
(1,243)\end{array}$ \\
\hline $\begin{array}{l}\text { Dificult. } \\
\text { económ. }\end{array}$ & $\begin{array}{l}-3,979 \\
(4,653)\end{array}$ & $\begin{array}{l}-1,048 \\
(4,950)\end{array}$ & $\begin{array}{l}-7,474^{\star *} \\
(3,653)\end{array}$ & $\begin{array}{l}-9,119^{\star \star} \\
(4,515)\end{array}$ & $\begin{array}{l}-4,906 \\
(3,939)\end{array}$ & $\begin{array}{l}5,089 \\
(5,130)\end{array}$ & $\begin{array}{l}-3,619 \\
(3,707)\end{array}$ & $\begin{array}{l}-9,030^{\star \star} \\
(4,664)\end{array}$ & $\begin{array}{l}-3,857^{\star \star \star} \\
(1,421)\end{array}$ \\
\hline $\begin{array}{l}\text { Nec. no } \\
\text { cumplidas }\end{array}$ & $\begin{array}{l}-13,198^{\star \star \star} \\
(4,938)\end{array}$ & $\begin{array}{l}-3,669 \\
(4,746)\end{array}$ & $\begin{array}{l}-4,927 \\
(4,147)\end{array}$ & $\begin{array}{l}0,071 \\
(4,197)\end{array}$ & $\begin{array}{l}0,994 \\
(4,099)\end{array}$ & $\begin{array}{l}-6,136 \\
(4,127)\end{array}$ & $\begin{array}{l}-3,947 \\
(3,746)\end{array}$ & $\begin{array}{l}-7,401^{*} \\
(4,706)\end{array}$ & $\begin{array}{l}-2,719^{*} \\
(1,426)\end{array}$ \\
\hline Se siente feliz & $\begin{array}{l}1,397^{\star *} \\
(0,576)\end{array}$ & $\begin{array}{l}1,286^{*} \\
(0,721)\end{array}$ & $\begin{array}{l}3,924^{\star \star \star} \\
(0,769)\end{array}$ & $\begin{array}{l}2,115^{\star \star \star} \\
(0,634)\end{array}$ & $\begin{array}{l}2,670^{\star \star \star} \\
(0,671)\end{array}$ & $\begin{array}{l}2,587^{\star \star \star} \\
(0,721)\end{array}$ & $\begin{array}{l}2,827^{\star \star \star} \\
(0,825)\end{array}$ & $\begin{array}{l}0,944 \\
(0,815)\end{array}$ & $\begin{array}{l}2,022^{\star \star \star} \\
(0,236)\end{array}$ \\
\hline Grupo discrim. & $\begin{array}{l}-1,985 \\
(5,399)\end{array}$ & $\begin{array}{l}-11,242^{\star *} \\
(5,076)\end{array}$ & $\begin{array}{l}-3,814 \\
(4,038)\end{array}$ & $\begin{array}{l}9,583^{*} \\
(5,044)\end{array}$ & $\begin{array}{l}-15,662^{\star \star \star} \\
(5,948)\end{array}$ & $\begin{array}{l}-6,594 \\
(4,333)\end{array}$ & $\begin{array}{l}-4,079 \\
(4,954)\end{array}$ & $\begin{array}{l}-9,416 \\
(7,320)\end{array}$ & $\begin{array}{l}-6,450^{\star \star \star} \\
(1,695)\end{array}$ \\
\hline $\begin{array}{l}\text { Frec. con. } \\
\text { fruta }\end{array}$ & $\begin{array}{l}0,530 \\
(1,075)\end{array}$ & $\begin{array}{l}2,431^{\star *} \\
(1,087)\end{array}$ & $\begin{array}{l}0,804 \\
(1,084)\end{array}$ & $\begin{array}{l}1,483 \\
(0,954)\end{array}$ & $\begin{array}{l}0,298 \\
(0,946)\end{array}$ & $\begin{array}{l}1,670 \\
(1,248)\end{array}$ & $\begin{array}{l}0,406 \\
(1,099)\end{array}$ & $\begin{array}{l}-0,254 \\
(1,101)\end{array}$ & $\begin{array}{l}0,745^{\star *} \\
(0,362)\end{array}$ \\
\hline $\begin{array}{l}\text { Frec. con. } \\
\text { verdura }\end{array}$ & $\begin{array}{l}1,332 \\
(1,212)\end{array}$ & $\begin{array}{l}-0,820 \\
(1,245)\end{array}$ & $\begin{array}{l}-0,731 \\
(1,293)\end{array}$ & $\begin{array}{l}-2,722^{* *} \\
(1,262)\end{array}$ & $\begin{array}{l}0,631 \\
(1,264)\end{array}$ & $\begin{array}{l}-2,302^{*} \\
(1,290)\end{array}$ & $\begin{array}{l}0,025 \\
(1,335)\end{array}$ & $\begin{array}{l}2,181^{*} \\
(1,315)\end{array}$ & $\begin{array}{l}0,067 \\
(0,428)\end{array}$ \\
\hline $\begin{array}{l}\text { Actividad } \\
\text { física }\end{array}$ & $\begin{array}{l}1,003^{\star \star} \\
(0,474)\end{array}$ & $\begin{array}{l}0,281 \\
(0,555)\end{array}$ & $\begin{array}{l}1,803^{\star \star \star} \\
(0,523)\end{array}$ & $\begin{array}{l}0,228 \\
(0,468)\end{array}$ & $\begin{array}{l}0,803^{\star \star} \\
(0,466)\end{array}$ & $\begin{array}{l}0,998^{*} \\
(0,526)\end{array}$ & $\begin{array}{l}1,641^{\star \star \star} \\
(0,562)\end{array}$ & $\begin{array}{l}0,873^{*} \\
(0,522)\end{array}$ & $\begin{array}{l}1,000^{\star \star \star} \\
(0,173)\end{array}$ \\
\hline IMC & $\begin{array}{l}-12,525 \\
(29,722)\end{array}$ & $\begin{array}{l}42,053 \\
(32,666)\end{array}$ & $\begin{array}{l}-2,513 \\
(45,662)\end{array}$ & $\begin{array}{l}7,129 \\
(29,963)\end{array}$ & $\begin{array}{l}9,781 \\
(34,542)\end{array}$ & $\begin{array}{l}-37,700 \\
(30,295)\end{array}$ & $\begin{array}{l}-42,401 \\
(32,136)\end{array}$ & $\begin{array}{l}-69,212^{\star \star} \\
(31,925)\end{array}$ & $\begin{array}{l}-23,439 \text { *** } \\
(0,959)\end{array}$ \\
\hline $\begin{array}{l}\text { Fuma (o a } \\
\text { fumado) }\end{array}$ & $\begin{array}{l}1,048 \\
(2,498)\end{array}$ & $\begin{array}{l}-0,166^{\star \star} \\
(2,669)\end{array}$ & $\begin{array}{l}-0,587 \\
(2,870)\end{array}$ & $\begin{array}{l}1,658 \\
(2,411)\end{array}$ & $\begin{array}{l}-5,262^{* \star} \\
(2,554)\end{array}$ & $\begin{array}{l}0,799 \\
(2,836)\end{array}$ & $\begin{array}{l}4,044 \\
(2,653)\end{array}$ & $\begin{array}{l}0,505 \\
(2,935)\end{array}$ & $\begin{array}{l}0,701 \\
(0,891)\end{array}$ \\
\hline $\begin{array}{l}\text { Frec. con. } \\
\text { alcohol }\end{array}$ & $\begin{array}{l}1,895^{\star \star \star} \\
(0,585)\end{array}$ & $\begin{array}{l}-0,807 \\
(0,716)\end{array}$ & $\begin{array}{l}1,031 \\
(0,752)\end{array}$ & $\begin{array}{l}0,480 \\
(0,549)\end{array}$ & $\begin{array}{l}0,656 \\
(0,583)\end{array}$ & $\begin{array}{l}0,605 \\
(0,609)\end{array}$ & $\begin{array}{l}0,378 \\
(0,668)\end{array}$ & $\begin{array}{l}0,383 \\
(0,668)\end{array}$ & $\begin{array}{l}0,672^{\star \star \star} \\
(0,213)\end{array}$ \\
\hline Asis. primaria & $\begin{array}{l}-0,770 \\
(2,730)\end{array}$ & $\begin{array}{l}-4,849 \\
(3,054)\end{array}$ & $\begin{array}{l}-0,862 \\
(3,041)\end{array}$ & $\begin{array}{l}-3,384 \\
(2,572)\end{array}$ & $\begin{array}{l}-4,181 \\
(2,717)\end{array}$ & $\begin{array}{l}-5,886^{\star} \\
(3,202)\end{array}$ & $\begin{array}{l}-0,020 \\
(2,800)\end{array}$ & $\begin{array}{l}-5,041^{*} \\
(2,954)\end{array}$ & $\begin{array}{l}-2,958^{\star \star \star} \\
(0,955)\end{array}$ \\
\hline Asis. urgencia & $\begin{array}{l}-5,559^{\star \star} \\
(2,668)\end{array}$ & $\begin{array}{l}-6,478^{\star \star} \\
(2,831)\end{array}$ & $\begin{array}{l}-0,306 \\
(2,928)\end{array}$ & $\begin{array}{l}-1,156 \\
(2,830)\end{array}$ & $\begin{array}{l}-4,207 \\
(2,821)\end{array}$ & $\begin{array}{l}-1,685 \\
(3,346)\end{array}$ & $\begin{array}{l}-2,805 \\
(2,851)\end{array}$ & $\begin{array}{l}-4,973 \\
(3,152)\end{array}$ & $\begin{array}{l}-3,102^{\star * \star} \\
(0,985)\end{array}$ \\
\hline Asis. especiliz. & $\begin{array}{l}-3,551 \\
(2,578)\end{array}$ & $\begin{array}{l}-4,439 \\
(2,958)\end{array}$ & $\begin{array}{l}1,799 \\
(2,674)\end{array}$ & $\begin{array}{l}-5,850^{\star \star \star} \\
(2,308)\end{array}$ & $\begin{array}{l}-1,917 \\
(2,677)\end{array}$ & $\begin{array}{l}-6,378^{\star *} \\
(3,090)\end{array}$ & $\begin{array}{l}-6,947^{\star \star *} \\
(2,768)\end{array}$ & $\begin{array}{l}-5,865^{\star \star} \\
(2,757)\end{array}$ & $\begin{array}{l}-4,579^{\star \star \star} \\
(0,927)\end{array}$ \\
\hline Edad & $\begin{array}{l}0,029 \\
(0,092)\end{array}$ & $\begin{array}{l}-0,456^{\star \star \star} \\
(0,103)\end{array}$ & $\begin{array}{l}-0,146 \\
(0,101)\end{array}$ & $\begin{array}{l}-0,263^{\star \star \star} \\
(0,089)\end{array}$ & $\begin{array}{l}-0,200^{\star \star} \\
(0,095)\end{array}$ & $\begin{array}{l}-0,112 \\
(0,103)\end{array}$ & $\begin{array}{l}-0,329^{\star \star \star} \\
(0,100)\end{array}$ & $\begin{array}{l}-0,151 \\
(0,116)\end{array}$ & $\begin{array}{l}-0,210^{\star \star \star} \\
(0,033)\end{array}$ \\
\hline Sexo (mujer) & $\begin{array}{l}3,803 \\
(2,760)\end{array}$ & $\begin{array}{l}-1,201 \\
(2,848)\end{array}$ & $\begin{array}{l}-1,186 \\
(3,396)\end{array}$ & $\begin{array}{l}-4,981^{*} \\
(2,628)\end{array}$ & $\begin{array}{l}-0,423 \\
(2,770)\end{array}$ & $\begin{array}{l}-8,255^{\star \star \star} \\
(2,785)\end{array}$ & $\begin{array}{l}-3,867 \\
(2,915)\end{array}$ & $\begin{array}{l}-9,042^{\star \star \star} \\
(3,044)\end{array}$ & $\begin{array}{l}-3,717^{\star \star \star} \\
(0,976)\end{array}$ \\
\hline Habitat & $\begin{array}{l}-0,547 \\
(1,100)\end{array}$ & $\begin{array}{l}-0,236 \\
(1,272)\end{array}$ & $\begin{array}{l}0,813 \\
(1,102)\end{array}$ & $\begin{array}{l}0,217 \\
(1,014)\end{array}$ & $\begin{array}{l}-0,949 \\
(1,088)\end{array}$ & $\begin{array}{l}0,135 \\
(1,348)\end{array}$ & $\begin{array}{l}0,046 \\
(1,262)\end{array}$ & $\begin{array}{l}-0,120 \\
(1,239)\end{array}$ & $\begin{array}{l}-0,136 \\
(0,375)\end{array}$ \\
\hline Const. & $\begin{array}{l}23,116^{\star} \\
(13,069)\end{array}$ & $\begin{array}{l}63,707^{\star \star \star} \\
(14,900)\end{array}$ & $\begin{array}{l}40,083^{\star \star \star} \\
(18,068)\end{array}$ & $\begin{array}{l}67,977^{\star \star \star} \\
(12,744)\end{array}$ & $\begin{array}{l}47,339^{\star \star \star} \\
(15,021)\end{array}$ & $\begin{array}{l}60,565^{\star \star \star} \\
(16,079)\end{array}$ & $\begin{array}{l}68,170^{\star \star \star} \\
(15,510)\end{array}$ & $\begin{array}{l}72,307^{\star * \star} \\
(14,996)\end{array}$ & $\begin{array}{l}58,831^{\star \star \star} \\
(4,901)\end{array}$ \\
\hline R2 & 0,54 & 0,46 & 0,50 & 0,45 & 0,49 & 0,55 & 0,56 & 0,56 & 0,40 \\
\hline
\end{tabular}

Notas: ${ }^{*}: p<0,05 ;{ }^{* *}: p<0,01 ;{ }^{* * *}: p<0,001$.

Fuente: elaboración propia a partir de los datos de la encuesta del Proyecto IMPACT-A.5. Conclusiones 
Como comenzábamos diciendo al inicio de este informe, las circunstancias sociales y económicas en las que vivimos determinan nuestra salud física y mental (Allen et al., 2014; Alvarez-Galvez et al., 2013, 2014; Bambra et al., 2010; Eikemo et al., 2008; Marmot y Allen, 2014; Wilkinson, 1997). A pesar de la dificultad para llegar a establecer la asociación entre los diferentes factores de tipo socioeconómico que han podido incidir en la salud de los andaluces durante el periodo de crisis, los datos analizados a lo largo de este trabajo nos han permitido identificar algunos de los determinantes que presentan una mayor relevancia en la explicación de los problemas de salud de la población andaluza.

A partir de los datos generados para Andalucía, podemos observar que la crisis ha tenido un importante impacto en el conjunto de la comunidad. Por esta razón, es probable que las peores condiciones de los grupos de menor estatus socioeconómico (aquellos con mayores tasas de paro, riesgo de pobreza, etc.) hayan favorecido el desarrollo de viejas y nuevas desigualdades de salud. Los datos ponen de manifiesto la existencia de importantes desigualdades de salud que aún persisten en esta comunidad, como por ejemplo aquellas asociadas a las diferencias de género, la edad, los ingresos 0 la educación entre otras, las cuales se encuentran interrelacionadas. Estas desigualdades podrían verse incrementadas en el futuro si no se toman medidas que favorezcan la igualdad entre los diferentes grupos sociales que presentan un mayor riesgo de padecerlas (Heras-Mosteiro et al., 2016). En Andalucía, como en el resto de España, se reproducen las desigualdades de salud habituales y persistentes dentro del ámbito nacional y europeo (Bambra, 2010; Mackenbach, 2012). Desigualdades por discriminación étnica, por género, por edad, desempleo, discapacidad y problemas de salud mental, que como hemos podido comprobar a partir del presente análisis se encuentran asociadas a los problemas socioeconómicos de las familias, sus circunstancias vitales y estilos de vida.

El sexo y la edad son dos claros determinantes de la salud que nos vienen dados, pero estos factores a su vez se encuentran vinculados a los determinantes estructurales de toda sociedad, así como a otros factores mediadores asociados a los estilos de vida. En este sentido, la relevancia del sexo 0 la edad en la determinación de la salud puede incrementarse en aquellas sociedades — como la española — con un elevado desempleo y mercado de trabajo duales en las cuales encontramos una línea divisoria entre insiders (trabajadores a tiempo completo, con amplias coberturas sociales y bien pagados) y outsiders (trabajadores a tiempo parcial generalmente ocupados por jóvenes, mujeres e inmigrantes, los cuales presentan un mayor riesgo de salir del mercado de trabajo) (Alvarez-Galvez et al., 2016). Los resultados del presente estudio indican que las mujeres tienen peor salud que los hombres, pero también los jóvenes parados que buscan una primera oportunidad tienen peor salud o incluso personas mayores que también encuentran dificultades para volver a entrar en el mercado de trabajo una vez que han salido.

La salud de los andaluces presenta una importante correlación con el nivel educativo, lo cual responde en gran medida a la pauta del conjunto de España (Alvarez-Galvez, 2016, 2014, 2013). Al tener un sistema sanitario público, las desigualdades de salud asociadas a factores exclusivamente económicos se ven notablemente reducidas, aunque claramente siguen teniendo un efecto importante entre los grupos socioeconómicos más vulnerables. En este sentido, aunque los ingresos no resultan un factor determinante de la salud de la población andaluza, sí demuestran un efecto indirecto en la salud y la calidad de vida a través de la reducción de las posibilidades económicas para adquirir determinados recursos sociales y sanitarios (medicamentos, tratamientos dentales, terapias, etc.). En todo caso, se hace patente la importancia de la educación dentro del contexto andaluz, no ya solo porque la educación es el motor del «ascensor social» que ofrece a los ciudadanos la posibilidad de crecer en términos laborales y personales, sino también como un medio fundamental de mejorar y conservar nuestra salud futura, así como la de las personas que nos rodean. Ahora bien, como se ha puesto de manifiesto recientemente, también es necesario buscar un encaje laboral adecuado a los niveles de formación, ya que fenómenos como la sobre-educación y la sobre-cualificación de los jóvenes acaban generando problemas de salud cuando las expectativas laborales de estos grupos no se ven cumplidas en el medio y largo plazo (Bracke, Pattyn y Knesebeck, 2013).

La educación es el canalizador de nuestra profesión futura, de nuestro nivel de ingresos, pero también de las opiniones y actitudes que mantenemos y nuestra forma de comportarnos, así como también de nuestras posibilidades para enfrentarnos a la vida ante cualquier contingencia. De ahí que, en los próximos años, para conservar la salud de nuestros ciudadanos y la calidad de nuestro sistema sanitario, no solo resultará necesario reactivar el gasto sanitario para reducir las actuales desigualdades de salud, sino también el gasto en educación y en políticas, laborales y sociales, que den soporte tanto a aquellos grupos que aún siguen atravesando dificultades socioeconómicas como a aquellos que puedan encontrarse en condiciones de vulnerabilidad y riesgo de padecer estos problemas en el futuro. Así, las actuaciones futuras para paliar los efectos de la crisis sobre la salud deberían ser implementadas desde una óptica integral que tenga en cuenta la compleja interrelación y mutabilidad de los determinantes sociales de la salud sobre los diferentes grupos de nuestra sociedad. 


\section{Bibliografía}

\section{ALLEN, J.; BALFOUR, R.; BELL, R. Y MARMOT, M. (2014):}

«Social determinants of mental health», Int. Rev. Psychiatry, 26, pp. 392-407.

\section{ALVAREZ-GALVEZ, J. (2016): \\ «Discovering complex interrelationships between socioeconomic status and health in Europe: A case study applying Bayesian Networks», Soc. Sci. Res., 56, pp. 133-143.}

\section{ÁLVAREZ-GÁLVEZ, J. (2016): \\ «Measuring the effect of ethnic and non-ethnic discrimination over Euro- peans' self-rated health, International Journal of Public Health», Internatio- nal Journal of Public Health, vol. 61, Issue 3, pp. 367-374.}

\section{ALVAREZ-GALVEZ, J.; RODERO-COSANO, M. L.; GARCÍA-ALONSO, C.} Y SALVADOR-CARULLA, L. (2014):

"Changes in socioeconomic determinants of health: comparing the effect of social and economic indicators through European welfare state regimes», $J$. Public Health (Bangkok), 22, pp. 305-311.

\section{ALVAREZ-GALVEZ, J.; RODERO-COSANO, M. L.; MOTRICO, E.;} SALINAS-PEREZ, J. A.; GARCIA-ALONSO, C. Y SALVADOR-CARULLA, L. (2013): «The impact of socio-economic status on self-rated health: study of 29 countries using European social surveys (2002-2008)», Int. J. Environ. Res. Public Health, 10, pp. 747-61.

\section{ALVAREZ-GALVEZ, J. Y SALVADOR-CARULLA, L. (2013):}

«Perceived discrimination and self-rated health in Europe: evidence from the European Social Survey (2010)», PLoS One, 8, e74252.

\section{BAMBRA, C.; LUNAU, T.; VAN DER WEL, K. A.; EIKEMO, T. A.} Y DRAGANO, N. (2014):

«Work, health, and welfare: the association between working conditions, welfare states, and self-reported general health in Europe», Int. J. Health Serv., 44, pp. 113-136.

\section{BAMBRA, C.; NETUVELI, G. Y EIKEMO, T. A. (2010):}

«Welfare state regime life courses: the development of western European welfare state regimes and age-related patterns of educational inequalities in self-reported health», Int. J. Health Serv., 40, pp. 399-420.
BARTLEY, M.; POWER, C.; BLANE, D.; SMITH, G. D. Y SHIPLEY, M. (1994): «Birth weight and later socioeconomic disadvantage: evidence from the 1958 British cohort study», BMJ, 309, pp. 1.475-1.478.

\section{BARTLEY, M.; SACKER, A. Y CLARKE, P. (2004):}

«Employment status, employment conditions, and limiting illness: prospective evidence from the British household panel survey 1991-2001», J. Epidemiol. Community Health, 58, pp. 501-506.

\section{BECKFIELD, J. Y KRIEGER, N. (2009):}

«Epi + demos + cracy: linking political systems and priorities to the magnitude of health inequities--evidence, gaps, and a research agenda», Epidemiol. Rev., 31, pp. 152-177<doi:10.1093/epirev/mxp002>.

\section{BENZEVAL, M. Y JUDGE, K. (2001):}

«Income and health: the time dimension», Soc. Sci. Med., 52, pp. 1.371-1.390.

\section{BLANE, D.; HARDING, S. Y ROSATO, M. (1999):}

«Does social mobility affect the size of the socioeconomic mortality differential?: evidence from the Office for National Statistics Longitudinal Study", J. R. Stat. Soc. Ser. A. Stat. Soc., 162, pp. 59-70.

\section{BORG, V. Y KRISTENSEN, T. S. (2000):}

«Social class and self-rated health: can the gradient be explained by differences in life style or work environment?», Soc. Sci. Med., 51, pp. 1.019-1.030.

\section{BRACKE, P.; PATTYN, E. Y VON DEM KNESEBECK, O. (2013):}

«Overeducation and depressive symptoms: diminishing mental health returns to education», Sociol. Health IIIn., 35, pp. 1.242-1.259.

\section{CASSEL, J. (1976):}

«The contribution of the social environment to host resistance: the Fourth Wade Hampton Frost Lecture», Am. J. Epidemiol., 104, pp. 107-123.

\section{CHUNG, H. Y MUNTANER, C. (2007):}

«Welfare state matters: a typological multilevel analysis of wealthy countries», Health Policy, 80, pp. 328-339.

\section{CHUNG, H.; NG, E.; IBRAHIM, S.; KARLSSON, B.; BENACH, J.;} ESPELT, A. Y MUNTANER, C. (2013):

«Welfare state regimes, gender, and depression: a multilevel analysis of middle and high income countries», Int. J. Environ. Res. Public Health, 10, pp. 1.324-1.341. 
DAVEY SMITH, G.; HART, C.; HOLE, D.; MACKINNON, P.; GILLIS, C.; WATT, G.; BLANE, D. Y HAWTHORNE, V. (1998):

"Education and occupational social class: which is the more important indicator of mortality risk?», J. Epidemiol. Community Health, 52, pp. 153-160.

\section{EIKEMO, T. A.; BAMBRA, C.; JOYCE, K. Y DAHL, E. (2008):}

«Welfare state regimes and income-related health inequalities: a comparison of 23 European countries», Eur. J. Public Health, 18, pp. 593-599.

\section{HART, C. L.; SMITH, G. D.; BLANE, D. (1998):}

«Social mobility and 21 year mortality in a cohort of Scottish men», Soc. Sci. Med., 47, pp. 1.121-1.130.

\section{HERAS-MOSTEIRO, J.; LEGIDO-QUIGLEY, H.; SANZ-BARBERO, B.} Y OTERO-GARCIA, L. (2016):

«Health Care Austerity Measures in Times of Crisis: The Perspectives of Primary Health Care Physicians in Madrid», Spain. Int. J. Health Serv.

\section{ILLSLEY, R. (1955):}

«Social class selection and class differences in relation to stillbirths and infant deaths», Br. Med. J., 2, pp. 1.520-1.524.

JAKAB, Z. Y MARMOT, M. (2014):

«Social determinants of health in Europe», Lancet, 379, pp. 103-105.

KAPLAN, G. A.; PAMUK, E. R.; LYNCH, J. W.; COHEN, R. D. YBALFOUR, J. L. (1996):

«Inequality in income and mortality in the United States: analysis of mortality and potential pathways», BMJ, 312, pp. 999-1.003.

\section{KRIEGER, N. (2005):}

«Embodiment: a conceptual glossary for epidemiology», J. Epidemiol. Community Health, 59, pp. 350-355.

\section{KRIEGER, N. (2001):}

«Theories for social epidemiology in the 21st century: an ecosocial perspective», Int. J. Epidemiol., 30, pp. 668-677.

\section{LAWRENCE, E. M.; ROGERS, R. G. Y WADSWORTH, T. (2015):}

«Happiness and longevity in the United States», Soc. Sci. Med., 145, pp. 115-119.

LOBMAYER, P. Y WILKINSON, R. G. (2002):

«Inequality, residential segregation by income, and mortality in US cities», J. Epidemiol. Community Health, 56, pp. 183-187.
LYNCH, J. W.; KAPLAN, G. A.; PAMUK, E. R.; COHEN, R. D.; HECK, K. E.; BALFOUR, J. L. Y YEN, I. H. (1998):

«Income inequality and mortality in metropolitan areas of the United States», Am. J. Public Health, 88, pp. 1.074-1.080.

LYNCH, J.; SMITH, G. D.; HILLEMEIER, M.; SHAW, M.;

RAGHUNATHAN, T. Y KAPLAN, G. (2001):

«Income inequality, the psychosocial environment, and health: comparisons of wealthy nations», Lancet, 358, Londres, pp. 194-200 <doi:10.1016/ S0140-6736(01)05407-1>.

\section{MACKENBACH, J. P. (2012):}

"The persistence of health inequalities in modern welfare states: The explanation of a paradox», Soc. Sci. Med., 75, pp. 761-769.

\section{MARMOT, M. (2002):}

"The influence of income on health: views of an epidemiologist», Health Aff. Millwood, 21, pp. 31-46.

\section{MARMOT, M. Y ALLEN, J. J. (2014):}

"Social determinants of health equity», Am. J. Public Health.

\section{MARMOT, M. Y WILKINSON, R. G. (2001):}

«Psychosocial and material pathways in the relation between income and health: a response to Lynch et al.», BMJ, 322, pp. 1.233-1.236.

\section{MUNTANER, C.; BORRELL, C.; NG, E.; CHUNG, H.; ESPELT, A.;} RODRIGUEZ-SANZ, M.; BENACH, J. Y O'CAMPO, P. (2011):

«Politics, welfare regimes, and population health: controversies and evidence», Sociol. Health IIIn., 33, pp. 946-964.

\section{NAVARRO, V. Y SHI, L. (2001):}

«The political context of social inequalities and health», Int. J. Health Serv., 31, pp. 1-21.

\section{POWER, C.; STANSFELD, S. A.; MATTHEWS, S.; MANOR, 0.}

Y HOPE, S. (2002):

"Childhood and adulthood risk factors for socio-economic differentials in psychological distress: evidence from the 1958 British birth cohort», Soc. Sci. Med., 55, pp. 1.989-2.004.

\section{RAPHAEL, D. (2006):}

«Social determinants of health: present status, unanswered questions, and future directions», Int. J. Health Serv., 36, pp. 651-677. 
RAPHAEL, D. Y BRYANT, T. (2006):

«Maintaining population health in a period of welfare state decline: political economy as the missing dimension in health promotion theory and practice», Promot. Educ., 13, pp. 236-242.

\section{SOLAR, O. Y IRWIN, A. (2007):}

"A conceptual framework for action on the social determinants of health", WHO Discussion.

\section{SONEGO, M.; LLACER, A.; GALAN, I. Y SIMON, F. (2013):}

"The influence of parental education on child mental health in Spain», Qual. Life Res., 22, pp. 203-211.

\section{WEST, P. (1991):}

"Rethinking the health selection explanation for health inequalities», Soc. Sci. Med., 32, pp. 373-384.

\section{WILKINSON, R. G. (1997):}

«Socioeconomic determinants of health. Health inequalities: relative or absolute material standards?», BMJ, 314, pp. 591-595.

\section{WILKINSON, R. G. Y PICKETT, K. E. (2006):}

«ncome inequality and population health: a review and explanation of the evidence», Soc. Sci. Med., 62, pp. 1.768-1.784.

\section{WHITEHEAD, M. (1992):}

«The concepts and principles of equity and health», Int. J. Health Serv., 22, pp. 429-445. 


\section{NÚMEROS PUBLICADOS}

\section{7}

Elecciones 2008 en Andalucía: concentración y continuidad

La medición del efecto de las externalidades del capital humano en España y Andalucía. 1980-2000

Protección legislativa del litoral andaluz frente a las especies invasoras: el caso Doñana

El valor monetario de la salud: estimaciones empíricas

La educación postobligatoria en España y Andalucía

La pobreza dual en Andalucía y España

Jubilación y búsqueda de empleo a edades avanzadas

El carácter social de la política de vivienda en Andalucía.

Aspectos jurídicos

El camino del éxito: jóvenes en ocupaciones de prestigio

Mutantes de la narrativa andaluza

Gobernanza multinivel en Europa. Una aproximación desde el caso andaluz

Partidos políticos, niveles de gobierno y crecimiento económico regional

Bilingüismo y Educación. Incidencia de la Red de Centros Bilingües de Andalucía

Marroquíes en Andalucía. Dinámicas migratorias y condiciones de vida

Obstáculos y oportunidades. Análisis de la movilidad social intergeneracional en Andalucía

El vandalismo como fenómeno emergente en las grandes ciudades andaluzas

Transformando la gestión de recursos humanos en las administraciones públicas

Valores y conductas medioambientales en España

¿Sabemos elegir? Introducción al estudio de la conducta económica de las personas

Metro ligero e innovación para la movilidad sostenible de las áreas metropolitanas andaluzas

El papel de las regiones en la actual Unión Europea

Nuevos enfoques en el diseño de los copagos farmacéuticos

La inmigración en Andalucía. Un análisis con datos de la Seguridad Social (2007-2008)

Arte contemporáneo y sociedad en Andalucía

La creación de una nueva realidad empresarial. El caso de Andalucía

Nuevos modelos de familia en Andalucía y políticas públicas

Rasgos básicos del envejecimiento demográfico y las personas mayores en Andalucía
Género, salud y orden social. El caso del modelo clínico de transexualidad

Gestión del pluralismo religioso en el ámbito autonómico y local

La educación como factor determinante de la movilidad intergeneracional en Andalucía

Las compañías de bajo coste en los aeropuertos andaluces La construcción del sujeto político entre los jóvenes en riesgo

La disposición a pagar por el medio ambiente. Un análisis con datos de Andalucía

0: La inmigración en Andalucía. Un análisis con datos de la Seguridad Social en 2009

Percepción de la desigualdad y demanda de políticas redistributivas en Andalucía

Las violencias masculinas y la prevención de la violencia contra las mujeres

62. Las violencias masculinas y la prevención de la violencia contra las mujeres

La población infantil ante las nuevas tecnologías de la información. Una aproximación a la realidad de los nativos digitales andaluces

El contacto de la ciudadanía con los ayuntamientos como forma de participación política en Andalucía

Hacia un modelo de movilidad urbana sostenible

Las transiciones hacia el empleo de la juventud andaluza

67: El sector de los alimentos ecológicos en Andalucía: diagnóstico. retos y estrategias

Percepción de los españoles y andaluces ante la pobreza

La presencia de las mujeres en los ayuntamientos andaluces (1979-2011)

Un relato sobre identidad y vida buena en Andalucía

Bienestar, desigualdad y pobreza en Andalucía: un estudio comparativo con el resto de España a partir de las encuestas de condiciones de vida 2006 y 2012

2: Competencias autonómicas y gestión de la cuenca del Guadalquivir

Reformas legislativas, incumplimientos de la Carta Social Europea y su invocación en los órganos judiciales

4: Reforma constitucional y nuevo paradigma del Estado social. De la ordenación contingente a la organización consciente del bienestar

Bullying, cyberbullying y dating violence. Estudio de la gestión de la vida social en estudiantes de Primaria y Secundaria de Andalucía

¿Odiamos la política?

Determinantes sociales de la salud en Andalucía 


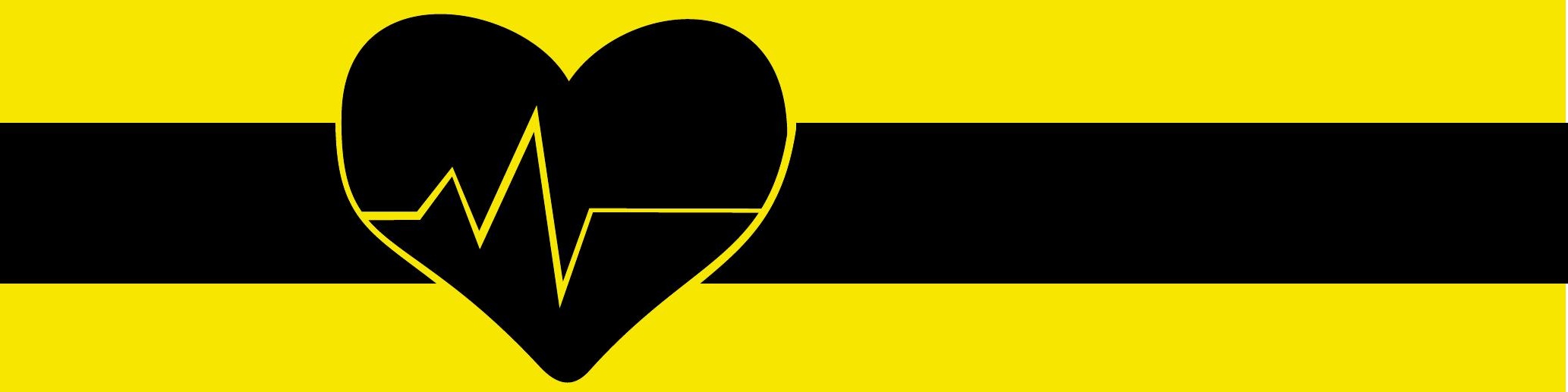

